Potential Improvements for HEC-HMS Automated Parameter Estimation

Brian E. Skahill 


\title{
Potential Improvements for HEC-HMS Automated Parameter Estimation
}

\author{
Brian E. Skahill
}

Coastal and Hydraulics Laboratory

U.S. Army Engineer Research and Development Center

3909 Halls Ferry Road

Vicksburg, MS 39180-6199

Final report

Approved for public release; distribution is unlimited. 


\begin{abstract}
Model independent enhancements and adaptations to the Gauss-Marquardt-Levenberg (GML) method of computer-based parameter estimation are described and demonstrated as potential improvements to existing HEC-HMS automatic calibration capabilities. In contrast to existing HEC-HMS automated parameter estimation capabilities, these methods support global optimization, the ability to simultaneously calibrate multiple subwatershed systems represented within an HEC-HMS model, and they also provide information about individual parameter sensitivities and parameter correlation during and at the end of the calibration process. Moreover, their model independent nature allows one to include into the calibration process (1) state information other than simply stream discharge data, (2) multiple periods, rather than a single time window, of the calibration dataset(s), and (3) the ability to weight data in order to accommodate a prediction specific calibration effort or to accommodate suspect and/ or missing observations. The methods are demonstrated by calibrating HEC-HMS models to subwatershed systems in the Goodwin Creek Experimental Watershed.
\end{abstract}

DISCLAIMER: The contents of this report are not to be used for advertising, publication, or promotional purposes. Citation of trade names does not constitute an official endorsement or approval of the use of such commercial products. All product names and trademarks cited are the property of their respective owners. The findings of this report are not to be construed as an official Department of the Army position unless so designated by other authorized documents. 


\section{Contents}

Figures and Tables......................................................................................................................

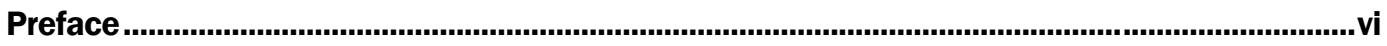

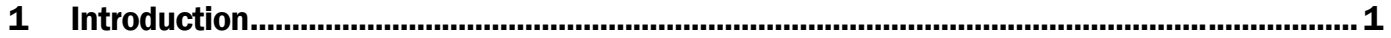

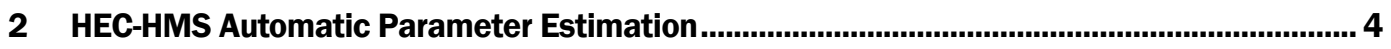

3 Potential Improvements for HEC-HMS Automated Parameter Estimation ............................. 8

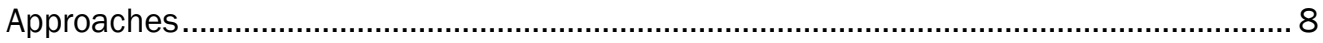

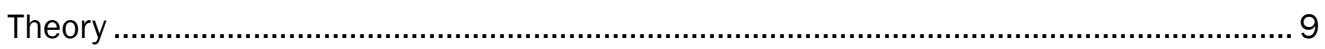

Gauss-Marquardt-Levenberg Parameter Estimation ......................................................... 9

The Trajectory Repulsion Scheme.....................................................................................13

Temporary Parameter Immobilization..................................................................................15

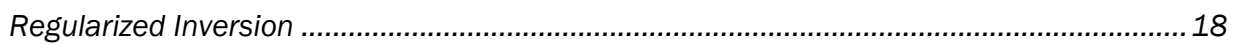

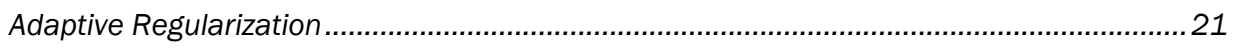

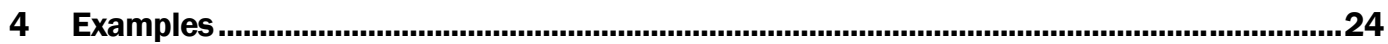

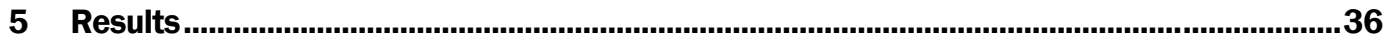

First HEC-HMS Model - Calibration Experiment 1 .........................................................36

First HEC-HMS Model - Calibration Experiment 2 .......................................................... 40

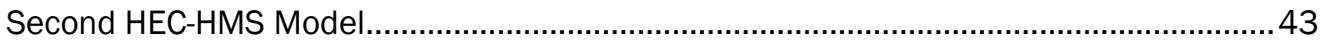

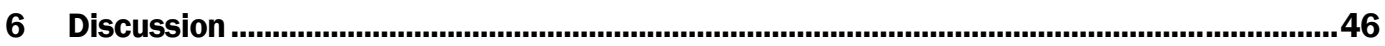

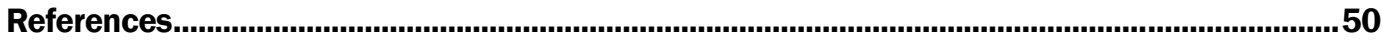

Report Documentation Page 


\section{Figures and Tables}

\section{Figures}

Figure 1. Goodwin Creek Experimental Watershed, delineated subwatersheds, derived stream network, rain and numbered streamflow gauge locations.

Figure 2. Plot of observed flow data used to calibrate the first HEC-HMS model.

Figure 3. Observed flow, quickflow, and base flow data at gauge 9 in Goodwin Creek Experimental Watershed.

Figure 4. Plot of station 8 observed flow data used to calibrate the second HMS model.

Figure 5. Plot of station 9 observed flow data used to calibrate the second HMS model.

Figure 6. Plot of station 11 observed flow data used to calibrate the second HMS model.............35

Figure 7. Plot of station 12 observed flow data used to calibrate the second HMS model.

Figure 8. End-points in parameter space of 100 inversion runs undertaken under the control of PD_MS2. Parameters comprising an optimized set are linked vertically between graphs by objective function. TPI functionality was not operative.

Figure 9. Simulated and observed flows at identified global minimum for the flows that were compared for the seventh non-contiguous time interval listed in Table 2.

Figure 10. Plot of simulated flows, quickflow, and base flow at the global minimum.

Figure 11. End-points in parameter space of 100 inversion runs undertaken under the control of PD_MS2. Parameters comprising an optimized set are linked vertically between graphs by objective function. TPI functionality was not operative.

Figure 12. Simulated and observed flows at identified global minimum for the flows that were compared for the seventh non-contiguous time interval listed in Table 2.

Figure 13. Plot of simulated flows, quickflow, and base flow at the global minimum.

Figure 14. Simulated and observed flows at station 9, at the minimum objective function value, for the flows that were compared for the seventh non-contiguous time interval listed in Table 2

Figure 15. Plot of simulated flows, quickflow, and base flow, for station 9, at the minimum.

\section{Tables}

Table 1. Four of the six objective function definitions available within HEC-HMS.

Table 2. Non-contiguous time intervals and thresholds, both which determined model to measurement misfit for both experiments with the first model.

Table 3. HEC-HMS parameters, their functions, and constraints imposed during the calibration process.

Table 4. For the second HEC-HMS model, non-contiguous time intervals and thresholds associated with station 8 .

Table 5. For the second HEC-HMS model, non-contiguous time intervals and thresholds associated with station 9 ...... 
Table 6. For the second HEC-HMS model, non-contiguous time intervals and thresholds associated with station 11 .

Table 7. For the second HEC-HMS model, non-contiguous time intervals and thresholds associated with station 12

Table 8. Nash-Sutcliffe efficiency associated with identified global minimum.

Table 9. Parameter values corresponding to global optimum; also shown are linear parameter confidence limits calculated as a by-product of the GML parameter estimation process.

Table 10. Initial parameter sets, final parameter sets, and objective function values associated with three HEC-HMS optimization runs with the first HEC-HMS model. 39

Table 11. Nash-Sutcliffe efficiency associated with identified global minimum.

Table 12. Estimated values for subwatershed model parameters for attainment of best fit at all subwatershed streamflow gauging stations, this corresponding to a measurement objective function of 135.1. Adaptive regularization was employed in the parameter estimation process.

Table 13. Number of terms and Nash-Sutcliffe efficiency scores, ES, associated with identified minimum for each station. 


\section{Preface}

This report was prepared by Brian E. Skahill, Watershed Systems Group, Coastal and Hydraulics Laboratory (CHL), U.S. Army Engineer Research and Development Center (ERDC), Vicksburg, MS.

Funding for this work was provided by the Flood and Coastal Storm Damage Reduction Research Program.

Daniel Christensen was a summer 2006 contract student in the Coastal and Hydraulics Laboratory at ERDC (ERDC-CHL), and is now a graduate student in the Department of Civil and Environmental Engineering at the University of Illinois at Urbana/ Champaign. Daniel assisted with the development of the HEC-HMS models that were used for the case study examples in this article. Daniel also performed the optimization runs for the first model within HEC-HMS. Dr. Bernard Hsieh, Hydrologic Systems Branch, ERDC-CHL, assisted by way of a literature review and summary related to the local search methods currently implemented within HECHMS.

This report was prepared under the general supervision of Earl V. Edris, Chief, Hydrologic Systems Branch, CHL; Dr. William D. Martin, Deputy Director, CHL; and Thomas W. Richardson, Director, CHL.

Colonel Richard B. J enkins was Commander and Executive Director of ERDC. The Director was Dr. J ames R. Houston. 


\section{Introduction}

Conceptual model structures for the continuous simulation of watershed hydrology are predefined, prior to modeling, by the hydrologist's understanding of the watershed system. With conceptual model structures, it is not possible to independently measure at least some of the model parameters; hence, they must be estimated through a formal model calibration exercise. Hence, the efficacy of a conceptual model structure to inform watershed management is heavily reliant upon observed system response data and the information that one can reliably glean from it during the calibration process.

Computer-based calibration of conceptual watershed model structures for continuous hydrologic simulation generally involves minimization of an objective function - a measure of model-to-measurement misfit. In simple cases this is comprised of differences between measured and modeled flows at, for example, daily, hourly, or even smaller intervals. In many cases, observed and modeled flows are transformed (for example through a Box-Cox transformation) before fitting, and/ or residuals are fitted to an ARMA model prior to formulation of an objective function, in order to reduce heteroscedascity and temporal correlation (Box and Tiao 1973, Box and J enkins 1976, Kuczera 1983, Bates and Campbell 2001). In more complex cases a multi-criteria objective function is constructed in which different measurement types, or the same measurement type processed in different ways, comprises separate components of a composite global objective function (Madsen 2000, Boyle et al. 2000, Doherty and Johnston 2003).

A unique solution to the inverse problem of model calibration can only be guaranteed if the number of parameters requiring estimation is commensurate with the information content of the calibration dataset. Often this is ensured by adherence to the so-called "principle of parsimony" in design of the inverse problem, in which parameters requiring adjustment through the calibration process are reduced to a number for which a unique estimate can be obtained for each. If calibration is computer-assisted, then prior to initiating execution of the parameter estimation software package, the modeler selects those parameters that he/ she wishes to estimate (normally on the basis of anticipated higher 
sensitivity of model outputs to these parameters) and holds other parameters fixed at "sensible values."

Often this method works well. However, problems associated with this approach include the following:

1. It is not always possible to know ahead of the parameter estimation process how many parameters can be estimated. If too few are selected for estimation it may not be possible to obtain a good fit between model outputs and field measurements. If too many are selected, the parameter estimation process may suffer from numerical instability and/ or result in the estimation of a set of parameter values that lack credibility.

2. Individual parameter sensitivities are not the sole determiner of what is estimable and what is not. Situations are often encountered where model outputs have a low sensitivity to certain parameters collectively, but can be very sensitive to the same parameters individually. This is the phenomenon of "parameter correlation."

3. Traditional approaches to calibration are not well suited to the solution of complex inverse problems, such as those involving simultaneous calibration of multiple models, where the estimation of useful values for otherwise nonuniquely estimable parameters may be assisted through the provision of trans-model parameter relationships, from which a departure will only be tolerated if supported by the calibration dataset.

These problems can be overcome through the use of parameter estimation algorithms that allow mathematical regularization to be implemented as part of the parameter estimation process itself. The result is a stable solution to the inverse problem (regardless of how ill-posed it is), and avoidance of the deleterious effects of numerical instability on both the parameter estimation process itself, and on the outcomes of that process, namely the set of estimated parameter values. A well-designed regularization algorithm, like its manual counterpart, achieves numerical stability by reformulating the inverse problem in a way that recognizes the level of parsimony that is necessary to attain a stable solution to that problem. However, this "parsimonizing" is undertaken in the context of a specific calibration dataset, allowing numerical stability to be achieved without 
compromising model-to-measurement fit any more than is deemed necessary by the modeler.

While measures can thus be taken to ensure mathematical tractability of an inverse problem posed on the basis of a properly-processed calibration dataset, it is rarely possible to avoid the fact that when calibrating conceptual watershed model structures the objective function will often contain local minima in addition to its global minimum (Wagener, Wheater, and Gupta 2004, and references cited therein). This presents challenges to the design of automatic calibration software, for a modeler who uses such software has the right to expect that estimated parameter sets result in the best possible fit between model outputs and field measurements (with due account taken of parameter believability).

The watershed model calibration concepts noted above will be explored while pursuing the objectives for this article which include describing and demonstrating the use of parameter estimation methodologies that could potentially be employed to improve upon existing HEC-HMS (Hydrologic Engineering Center's Hydrologic Modeling System) automated parameter estimation capabilities. In particular, the intent for this article is to describe and demonstrate the use of methods that (1) accommodate local minima (Skahill and Doherty 2006) and (2) support the inversion of complex (i.e., highly parameterized) models (Doherty and Skahill 2006), and through this process to also:

a. Demonstrate that each of these two methods provide information about individual parameter sensitivities and parameter correlation.

b. Demonstrate how state information other than stream discharge data alone can be included into the automatic calibration process of a HECHMS model.

c. Demonstrate how objective function definitions associated with specific periods of the calibration dataset can be included into the automatic calibration process of a HEC-HMS model. 


\section{HEC-HMS Automatic Parameter Estimation}

HEC-HMS is a widely used graphically-based integrated watershed modeling environment with a user interface to a collection of potential methods for computing excess precipitation, runoff transformation, and hydrologic routing, among others, to support event-based or continuous simulation of the hydrologic response of a dendritic watershed system subject to meteorological forcing. The graphical user interface enables seamless movement between the database, model development, computational engine(s), and post-processing capabilities of HEC-HMS.

Existing HEC-HMS automatic calibration capabilities include the ability to specify one of six different objective functions and one of two different search algorithms (HEC 2005). Four of the six objective function definitions currently available within HEC-HMS are summarized in Table 1 (HEC 2000).

Table 1. Four of the six objective function definitions available within HEC-HMS.

\begin{tabular}{|l|l|}
\hline Description & Equation \\
\hline 1-norm & $\Phi=\sum_{i=1}^{i=n}\left|Q_{o}(i)-Q_{s}(i)\right|$ \\
\hline 2-norm squared & $\Phi=\sum_{i=1}^{i=n}\left[Q_{o}(i)-Q_{s}(i)\right]^{2}$ \\
\hline Percent error in peak & $\Phi=100\left|\frac{Q_{s}(\text { peak })-Q_{o}(\text { peak })}{Q_{o}(\text { peak })}\right|$ \\
\hline $\begin{array}{l}\text { Peak-weighted root } \\
\text { mean square error }\end{array}$ & $\Phi=\left\{\frac{1}{n}\left[\sum_{i=1}^{i=n}\left(Q_{o}(i)-Q_{s}(i)\right)^{2}\left(\frac{Q_{o}(i)+Q_{o}(\text { mean })}{2 Q_{o}(\text { mean })}\right)\right]\right\}^{1 / 2}$ \\
\hline
\end{tabular}

$\Phi=$ objective function; $n=$ number of hydrograph ordinates; $Q_{o}=$ observed flows; $Q_{s}=$ simulated flows; $Q_{0}($ peak $)=$ observed peak; $Q_{s}($ peak $)=\operatorname{simulated~peak;~and~} Q_{o}($ mean $)=$ mean of observed flows. 
The remaining two objective function definitions currently available within HEC-HMS include the percent error in volume objective function and the time-weighted objective function (HEC 2005). Within HEC-HMS, the chosen objective function is evaluated for a single specified period of time.

The limited practical guidance provided in HEC (2005) for the selection of a specific objective function definition is supported by unrelated research. Conceptual watershed model structural uncertainty, due to simplifications and/ or inadequacies in hydrologic process descriptions, often results in a model's inability to fit all response modes of the hydrograph with a unique parameter set (Wagener, Wheater, and Gupta 2004, and references cited therein). Moreover, the predictive error variance analysis of Moore and Doherty (2005) indicated that model calibration and model prediction should not be treated as independent processes.

The two search algorithms within HEC-HMS include the univariategradient search algorithm and the derivative-free minimization algorithm of Nelder and Mead (1965).

The univariate-gradient local search algorithm is implemented within HEC-HMS in the following manner

$$
\begin{gathered}
x^{k+1}=x^{k}+\Delta x^{k} \\
\Delta x^{k}=-f^{\prime}\left(x^{k}\right) / f^{\prime \prime}\left(x^{k}\right)
\end{gathered}
$$

where $\mathrm{x}, \Delta x^{k}$, and $\mathrm{f}$ represent the adjustable model parameter, the parameter upgrade for $\mathrm{x}$, and the objective function, respectively (HEC 2000). HEC-HMS approximates the derivatives in Equation 2 numerically using finite difference methods. If the number of adjustable model parameters is greater than one, this procedure is applied successively to each adjustable parameter while holding all others constant.

The Nelder and Mead (1965) local search algorithm is a widely used derivative-free minimization algorithm that works in multiple dimensions. It is sometimes referred to as an "amoeba" method because it works by setting up rules that allow a cloud of points in parameter space to "crawl" to an objective function minimum in a vaguely amoeboid fashion. Rather than starting with a single initial guess for the optimized model, as with 
the univariate-gradient search algorithm, the Nelder and Mead method begins by selecting $m+1$ parameters which form a simplex - the simplest possible shape in an $\mathrm{m}$-dimensional parameter space. With the Nelder and Mead simplex algorithm, the $m+1$ vertices of a simplex of approximation to an optimal point in $\mathrm{m}$-dimensional parameter space are sampled, ordered by objective function value, and an attempt made to replace the worst vertex by reflection through the convex hull of the remaining vertices using limited sampling along the search direction so defined.

HEC-HMS supports constrained optimization for both search methods. HEC (2000) provides further details regarding the implementation of the two search algorithms within HEC-HMS.

Some limitations associated with the existing HEC-HMS automatic parameter estimation capabilities include the following:

1. Existing search methods within HEC-HMS are local; i.e., they suffer from the drawback that they may become trapped in local objective function minima, and thus report "optimized" parameter values that are not, in fact, optimized at all. This can seriously degrade their utility in the calibration of watershed models where local optima abound. Local search methods depend upon the initial model estimate. While one may attempt to rely on a judicious initial guess based on physical reasoning wherein adjustable model parameters are derived from watershed properties such as soils, this approach suffers from the drawback that the lumped conceptual model parameters are estimated based on point samples analyzed at the laboratory scale, and it requires further corroboration (Wagener, Wheater, and Gupta 2004, and references cited therein).

2. Within HEC-HMS, the calibration of multiple adjacent gauged subwatersheds currently requires one to calibrate each subwatershed model independently of the others rather than, for example, calibrating each model individually, with due recognition of the desirability of inter-subwatershed parameter similarity (i.e., parameter values in adjacent areas that are associated with similar physiographic features relevant to hydrologic response be at least broadly similar).

3. The current methods within HEC-HMS are not able to report useful information on parameter uncertainty, correlation, and sensitivity as a 
by-product of their use during and after the parameter estimation process.

4. Objective functions can only be comprised of differences between measured and modeled flows (i.e., inability to construct a multi-criteria objective function in which different measurement types (e.g., river stages, reservoir storages, evapotranspiration, snow water equivalent), or the same measurement type processed in different ways (e.g., flow, baseflow, quickflow, volume aggregations), comprise separate components of a composite global objective function).

5. Objective functions can only consist of a single time window for comparing differences between measured and modeled flows. In addition, there is no way to weight data, say for example, to guide a prediction specific calibration effort (Moore and Doherty 2005) or to accommodate suspect and/ or missing observations. 


\section{Potential Improvements for HEC-HMS Automated Parameter Estimation}

\section{Approaches}

Enhancements (Skahill and Doherty 2006) and adaptations (Doherty and Skahill 2006) to the Gauss-Marquardt-Levenberg (GML) method of computer-based parameter estimation (Levenburg 1944, Marquardt 1963), and a model independent protocol (Skahill 2006) wherein the inversion methods communicate with a model through the model's own input and output files, are presented as approaches to address the above noted limitations associated with existing HEC-HMS automatic parameter estimation capabilities.

Gradient-based methods such as the GML method have been criticized for poor performance in the face of local optima (Gupta et al. 2003). Use of such methods can lead to the determination of a parameter set that corresponds to a local, rather than global, objective function minimum, leaving the user with no idea of whether another location exists within parameter space for which the objective function is lower. Nevertheless, the method also has advantages, chief among these being its model-run efficiency, and its ability to report useful information on parameter sensitivities and covariances as a by-product of its use. It is also easily adapted to maintain this efficiency in the face of potential numerical problems (that adversely affect all parameter estimation methodologies) caused by parameter insensitivity and/ or parameter correlation.

The present article presents two algorithmic enhancements to the GML method that retain its strengths, but which overcome its weaknesses in the face of local optima. Using the first of these methods, an "intelligent search" for better parameter sets is conducted in parameter subspaces of decreasing dimensionality when progress of the parameter estimation process is slowed either by numerical instability incurred through problem ill-posedness, or when a local objective function minimum is encountered. The second methodology minimizes the chance of successive GML parameter estimation runs finding the same objective function minimum by starting successive runs at points that are maximally removed from previous parameter trajectories. As well as enhancing the ability of a GML- 
based method to find the global objective function minimum, the latter technique can also be used to find the locations of many non-global optima (should they exist) in parameter space. This can provide a useful means of inquiring into the well-posedness of a parameter estimation problem, and for detecting the presence of bimodal parameter and predictive probability distributions.

Moreover, the present article describes algorithmic adaptations to the GML method based on an efficient and stable mathematical regularization scheme. This scheme is a variant of so-called "Tikhonov regularization" in which the parameter estimation process is formulated as a constrained minimization problem. Use of the methodology eliminates the need for a modeler to formulate a parsimonious inverse problem in which a handful of parameters are designated for estimation prior to initiating the calibration process. Instead, the level of parameter parsimony required to achieve a stable solution to the inverse problem is determined by the inversion algorithm itself. Where parameters, or combinations of parameters, cannot be uniquely estimated, they are provided with values, or assigned relationships with other parameters, that are decreed to be realistic by the modeler. Conversely, where the information content of a calibration dataset is sufficient to allow estimates to be made of the values of many parameters, the making of such estimates is not precluded by "preemptive parsimonizing" ahead of the calibration process.

\section{Theory}

\section{Gauss-Marquardt-Levenberg Parameter Estimation}

Let the action of a model under calibration conditions be described by the model operator $\mathcal{M}$ that maps m-dimensional parameter space to the space of the $n$ observations that are available for use in the calibration process. Let the $\mathrm{m}$-dimensional vector $\mathrm{p}$ represent model parameters and the $\mathrm{n}$ dimensional vector $h$ represent observations. In many instances of watershed hydrologic model calibration these observations will represent stream discharges which have been "processed" in some way in order to achieve homoscedascity, and statistical independence of measurement "noise." The former is often achieved through a Box-Cox transformation (Box and Cox 1964), while the latter is often attempted through fitting residuals to an ARMA model, often as part of the parameter estimation process itself (Box and J enkins 1976, Kuczera 1983). The observations $h$ can be comprised of a single observation type, multiple observation types, 
and/ or a single observation type processed in different ways in order to ensure that the information content associated with different aspects of the calibration dataset exercise sufficient influence in the estimation of a final set of model parameters (Madsen 2000, Boyle et al. 2000, Doherty and J ohnston 2003).

Model calibration seeks to minimize some measure of model-tomeasurement misfit encapsulated in a "measurement objective function," herein designated as $\Phi_{\mathrm{m}}$. In the present instance this is defined as:

$$
\Phi_{\mathrm{m}}=[\mathcal{M}(\mathrm{p})-\mathrm{h}]^{\mathrm{t}} \mathrm{Q}[\mathcal{M}(\mathrm{p})-\mathrm{h}]
$$

where Q is a "weight matrix" which, in the context of watershed model calibration where $\mathrm{n}$ is large, is mostly comprised of diagonal elements only. Ideally, each diagonal element of Q is proportional to the inverse of the squared potential error associated with the corresponding processed measurement.

Where $p$ is estimable (i.e., where minimization of $\Phi_{\mathrm{m}}$ results in a unique parameter set), it is calculated as:

$$
p-p_{0}=\left(X^{t} Q X\right)^{-1} X^{t} Q\left(h-h_{0}\right)
$$

where $\mathrm{X}$ is the model J acobian matrix, each row of which is comprised of the derivatives (i.e., sensitivities) of a particular model output (for which there is a corresponding field measurement) with respect to all elements of p. These sensitivities are calculated at current parameter values, represented by $\mathrm{p}_{0}$, for which corresponding model outputs are $\mathrm{h}_{0}$. Where the model is nonlinear, p calculated through Equation 4 is not optimal (i.e., it does not minimize $\Phi_{\mathrm{m}}$ ) unless $\mathrm{p}_{0}$ is close to optimal. Hence, after Equation 4 is used to calculate an improved parameter set, a new set of sensitivities (i.e., $\mathrm{X}$ ) is calculated on the basis of the new parameter set, and the process is repeated until convergence to the objective function minimum is achieved.

In practice, the $\mathrm{X}^{\mathrm{t}} \mathrm{QX}$ matrix of Equation 4 is supplemented by addition of a diagonal term - the so-called "Marquardt lambda." Thus, Equation 4 becomes:

$$
p-p_{0}=\left(X^{t} Q X+\lambda I\right)^{-1} X^{t} Q\left(h-h_{0}\right)
$$


Normally $\lambda$ is adjusted during each iteration of the parameter estimation process such that its current value results in maximum parameter improvement during that iteration. When $\lambda$ is high it is easily shown that the direction of parameter improvement is the negative of the gradient of $\Phi_{\mathrm{m}}$, and under these conditions Equation 5 becomes equivalent to the "steepest descent" method of parameter estimation. While this method can result in rapid parameter improvement when parameters are far from optimal, its performance is disappointing in the vicinity of the objective function minimum, especially where that minimum occupies a long valley in parameter space as a result of excessive parameter correlation or insensitivity. In these circumstances "hemstitching" is likely to occur, where successive parameter improvements result in oscillations across the objective function valley, which is never actually penetrated. Hence, ideally $\lambda$ should commence the parameter estimation process with a moderate value, and then be reduced as the process progresses. However, if $\mathrm{X}^{\mathrm{t}} \mathrm{QX}$ is ill-conditioned, reducing the value of $\lambda$ will incur numerical instability as $\mathrm{X}^{\mathrm{t}} \mathrm{QX}+\lambda \mathrm{I}$ of Equation 5 is inverted. Hence, the Marquardt lambda has a secondary role, this being that of a de facto regularization device, with its value often being raised in order to prevent instability in the calculation of the parameter upgrade vector $\mathrm{p}-\mathrm{p}_{0}$. However, while the use of a high Marquardt lambda can prevent a relatively ill-posed parameter estimation problem from foundering, it achieves this at a cost in efficiency, for parameter upgrades become smaller at higher values of $\lambda$ as an inspection of Equation 5 suggests. Furthermore, as stated above, the ability of the calibration process to penetrate an elongate valley in parameter space may be severely compromised.

The predisposition of a matrix to stable inversion is often measured by its "condition number." High condition numbers result in amplification of numerical noise during the inversion process (Conte and de Boor 1972), while low condition numbers indicate that inversion should be possible with little numerical difficulty. In general, condition numbers for $\mathrm{X}^{\mathrm{t}} \mathrm{QX}$ greater than about $10^{4}$ are to be avoided, for at this level the numerical noise incurred through finite difference-based derivatives calculation for filling of the X matrix is amplified to the extent that parameter upgrades may lack integrity. While a raised Marquardt lambda can often rescue such a damaged process from total failure as described above, efficiency of the parameter estimation process is likely to be seriously degraded. 
Another problem that can be encountered when parameter estimation is accomplished by iterative calculation of $\mathrm{p}-\mathrm{p}_{0}$, using Equation 5 is that this process can converge to a parameter set $\mathrm{p}$ that corresponds to a local, rather than the global, minimum of the objective function. "Gradient methods," such as the GML method described above, that rely on equations such as Equation 5 have been criticized for this reason, and so-called "global search" methods such as SCE-UA (Duan et al. 1992) are often used instead. While a well-designed and robust global search method can indeed be guaranteed to minimize the objective function in spite of the existence of local minima, such robustness comes at a price, this being the high number of model runs that is normally required for completion of the parameter estimation process. To make matters worse, the number of model runs increases dramatically as the number of parameters requiring estimation increases. Use of Equation 5, on the other hand, is very runefficient. Fortunately, its propensity to find local minima can be mitigated through the use of schemes such as that described by Skahill and Doherty (2006) which combine the efficiency of gradient methods with the benefits of introducing a small degree of randomness to the parameter estimation process, together with an ability to "learn from past mistakes." In addition, Equation 5 can be enhanced by the inclusion of a regularization term (much more powerful than the Marquardt lambda as will be described shortly) that greatly increases the propensity for robust and efficient behavior when the dimension $\mathrm{m}$ of $\mathrm{p}$ is large, and the shape of the objective function surface in parameter space becomes a valley (or series of valleys) rather than a bowl (or series of bowls).

Gradient-based methods such as the GML method have been criticized for poor performance in the face of local optima (Gupta et al. 2003). Use of such methods can lead to the determination of a parameter set that corresponds to a local, rather than global, objective function minimum, leaving the user with no idea of whether another location exists within parameter space for which the objective function is lower. However certain features of the GML method make it difficult to reject outright as a serious contender for use in watershed model calibration. These features include the following:

1. In calibration contexts where local optima are rare or nonexistent, the GML method can normally find the objective function minimum in far fewer model runs than any other method. 
2. Estimates of parameter uncertainty, correlation and (in)sensitivity are readily available as a by-product of its use.

3. In cases of high parameter insensitivity and correlation, the method can be readily modified by the inclusion of various regularization devices to maintain numerical stability and robustness.

4. Various enhancements can be made to the GML method that allow it to carry out linear or nonlinear post-calibration predictive uncertainty analysis, with run efficiencies that far exceed those of MCMC methods (Vecchia and Cooley 1987).

It follows that if a methodology can be found that retains the advantages of the GML method, while eradicating its propensity to be trapped in local optima, such a method would deserve serious consideration for use in watershed model calibration.

\section{The Trajectory Repulsion Scheme}

The robust performance of the SCE-UA method, as well as that of most other global search methods, is based on two principles. These are as follows:

1. The injection of a certain degree of randomness into the parameter estimation process allows it to go in directions that may eventually prove fruitful, even if the attractiveness of a new direction may be shielded by the promise of local, more immediate, rewards.

2. The benefits of randomness are partly offset by the cost of making mistakes. Hence, by incorporating into a global optimization process an ability to learn from mistakes, the likelihood of incurring large runtime penalties through repeatedly making the same (or a similar) mistake is minimized.

Based on these principles, a modified form of the GML method was developed in order to increase the capacity of this method to work well in contexts where local minima occur. The package takes the form of a driver, in which GML parameter estimation is still conducted, but in which successive inversion runs are undertaken under intelligent control. The package is presently named "PD_MS2" (Skahill and Doherty 2006). 
PD_MS2 commences execution by running the model that it must calibrate $\mathrm{N}$ times, where $\mathrm{N}$ is set by the user. Experience has shown that between the square and the cube of the number of parameters requiring estimation is a suitable value for N. PD_MS2 employs random parameter values for these runs; these are sampled from a uniform or log-uniform distribution defined between user-supplied upper and lower parameter bounds.

PD_MS2 next ranks the outcomes of the $\mathrm{N}$ random runs in order of increasing objective function value. It then disregards all runs for which the objective function is above the median. Next, it initiates an inversion run, with initial values for this run being equal to the random parameter sample for which the objective function was lowest. PD_MS2 monitors this run, recording optimized parameter values, as well as parameter values calculated during every iteration of the nonlinear GML method which it implements. Normally between 5 and 15 such iterations are required to reach an objective function minimum. Each such iteration requires that at least as many model runs be undertaken as there are parameters requiring estimation, plus a few more.

After completion of the first inversion run, another inversion run is initiated. For this run it is desired that the chances of finding the same objective function minimum as that which was encountered on the first inversion run be minimized. Hence, from among the N/ 2 retained precalibration samples of parameter space, a starting point is chosen that is maximally distant from any point on the parameter trajectory taken by the initial inversion run. Selection of such a starting point is based on the rationale that the closer is a point in parameter space to the previous parameter trajectory, the more likely it is to lie in the "catchment area" of the previously-encountered objective function minimum.

After the next inversion run is complete, another parameter set is selected from the N/ 2 potential starting points. The parameter set selected is that which is maximally distant from all previous points on all previous trajectories. The process is then repeated.

A number of criteria can be used to terminate the PD_MS2 global optimization process. Where model run efficiency is an issue, PD_MS2 can be instructed to cease execution if the objective function has not been lowered over the last $\mathrm{M}_{1}$ inversion runs. Alternatively, PD_MS2 can be 
asked to undertake $\mathrm{M}_{2}$ inversion runs regardless of the outcomes of these runs. If $\mathrm{M}_{2}$ is moderate to large, this enables PD_MS2 to find the locations of many local optima in parameter space (should these exist), thus providing the user with powerful insights into the structure of the objective function surface.

It is worth noting that, as well as providing insights into the "broadscale" structure of the objective function response surface, PD_MS2 provides insights into the structure of this surface in the vicinity of the global objective function minimum as well. As has already been mentioned, the GML method can provide parameter sensitivities and can calculate a linear approximation to the parameter covariance matrix, as well as statistics derived from this matrix including correlation coefficients and eigenvectors/ eigenvalues of the covariance matrix. Information of this type is forthcoming only with difficulty from global search methods, this difficulty increasing with the number of parameters being estimated and with the degree of correlation between them (which, unfortunately, is the very situation in which such information is of most value).

\section{Temporary Parameter Immobilization}

"Temporary parameter immobilization" can be used as both a regularization device and as a device for conducting ordered attempts to break out of local pits in parameter space. This scheme is implemented only if the objective function improvement attained during a particular iteration of the GML process is less than a user-supplied threshold (normally 10 percent). In implementing this scheme, the most insensitive parameter is selected, and temporarily removed from the optimization process. With the dimensionality of estimable parameter space thus reduced (and with the most troublesome parameter being temporarily removed from the parameter estimation process), the parameter upgrade vector (which now has no component in the subspace of parameter space occupied by the temporarily frozen parameter) is recalculated using Equation 5. A model run is then conducted on the basis of the trial parameter set thus calculated in order to compute the objective function associated with this parameter set. Unless the objective function has fallen by a significant amount, the next most troublesome parameter is temporarily frozen (in addition to the first), and the parameter upgrade calculation procedure is repeated. After a number of parameters have been successively frozen in this manner (with already frozen parameters maintained in their frozen state), the process is abandoned, and then recommenced using a different value of 
the Marquardt lambda. For a parameter estimation problem involving $\mathrm{m}$ parameters, up to half of these parameters may be progressively frozen for up to three Marquardt lambdas, this requiring $3 \mathrm{~m} / 2$ model runs for that iteration for the testing of parameter upgrade vectors in addition to the (depending on whether forward differences or central differences are employed) $\mathrm{m}$ or $2 \mathrm{~m}$ model runs required for filling of the J acobian matrix. (Note, however, that the process is immediately abandoned if a suitable objective function improvement is obtained.) Thus, implementation of the TPI process may lead to the requirement that between twice and three times (at the very most) the number of model runs be carried out compared to normal GML operations. However, experience has demonstrated that on most occasions in which the TPI method is employed about 50 percent extra model runs need to be carried out, and that this is generally a small price to pay for the benefits that it brings in terms of increased numerical stability in situations of parameter nonuniqueness, and for a dramatic reduction in the risk of becoming trapped in local objective function pits.

The decreased probability of ensnarement in local optima that attends use of the TPI scheme has its roots in a number of properties of this scheme. One obvious reason for a heightened probability of success in finding its way out of small regions of attraction of limited extent in parameter space is the sheer number of parameter upgrades that are attempted by this scheme, together with the fact that the directions pertaining to these upgrade attempts tend to be maximally different with respect to each other. This maximality of difference is a result of two factors. The first is the fact that the upgrade direction tends to be dominated by insensitive parameters where all parameters are involved in the computation of this direction; this is a direct result of the fact that, because of their insensitivity, the GML parameter estimation algorithm calculates that these parameters require larger movement than other parameters to affect the objective function. As dimensions of parameter space are progressively closed to the parameter upgrade vector through the temporary immobilization of insensitive parameters, and new upgrade directions are accordingly computed in spaces of lower dimensions, these new directions will tend to be orthogonal to the original upgrade vector which was dominated by the now-omitted dimensions. The penchant for orthogonality is further increased as a result of the fact that the entire dimensionality reduction process is repeated for widely different Marquardt lambda values. As documented in works such as Bard (1974), computed upgrade directions 
can vary between that of steepest descent down the objective function surface when the Marquardt lambda is high, to a direction that can be almost orthogonal to this when the Marquardt lambda is low.

Another important factor behind the success of the TPI scheme is that it lowers the chances of upgraded parameters finding local optima in the first place. Unless objective function improvement during a particular iteration is acceptably large without the help of the TPI scheme (which often occurs in the early stages of the parameter estimation process), use of the TPI scheme requires that model runs be carried out specifically to test the ability of different upgrade vectors (often with very different directions as discussed above) to lower the objective function. The upgrade vector that results in the largest objective function decline is that which is selected as the basis for the next linearization of the inverse problem. Of all the upgrade vectors tested, this is the one least likely to lead to a local objective function minimum, for the encroachment of global or local optimality (for which derivatives of the objective function with respect to all model parameters is zero) is normally marked by smaller and smaller declines in the objective function per iteration as the GML method ensures that a parameter set is found from which all directions lead uphill. In fact, the more nonlinear is the problem, the less likely it is that a parameter upgrade vector resulting in a large objective function decline will lead directly to the bottom of an objective function minimum (due to the fact that the equations upon which this upgrade vector are calculated are based on an assumption whose inapplicability grows with increasing parameter movement, and/ or increasing changes in model outputs on account of this movement).

An additional factor that contributes to the success of the TPI scheme in both avoidance of local minima of small lateral extent, and in extricating itself from such minima, is use of finite differences for parameter derivatives calculation. As was mentioned above, parameter increments of one percent are often employed for forward difference derivatives calculation and two percent for central difference derivatives calculation. These increments are large enough to "see" outside of a small pit in which it may be currently trapped. Alternatively, if current parameter values lie just outside of a small pit, these increments are large enough for the effect of the pit to exert a smaller influence on calculated derivatives than would be the case if derivatives were exact. Thus, the use of finite-difference-based parameter derivatives provides a kind of filtering mechanism through which 
finer details of the objective function surface are prevented from concealing the broader features of that surface.

So, through a combination of the fact that many upgrade vectors are tested, that a parameter upgrade selection procedure is adopted that minimizes the chances of being trapped in a local minimum in the first place, and maximizes the chances of escaping from that minimum if ensnarement does indeed occur, and because parameter upgrades possess some immunity to the effects of pits because their calculation is based on finite-difference derivatives rather than point derivatives, use of the TPI method in calibration of surface water models has consistently resulted in good performance in estimating parameters for those models.

(Note that selection of a TPI activation threshold of 10 percent improvement in the objective function is somewhat arbitrary. However, experience has demonstrated that this normally results in efficient implementation of the method. If the threshold is set too high, TPI-based parameter upgrade recomputation will be undertaken on most GML optimization iterations, irrespective of proximity, or otherwise, to an objective function minimum. This can result in wasted model runs if rapid objective function improvement is taking place without the need for TPI upgrade repetitions. On the other hand, if the improvement threshold is set too low, then needless "struggling" of the GML method in the face of difficulties incurred through problem ill-posedness or proximity to a local minimum, resulting in only small improvements in the objective function in successive iterations, can be avoided.)

\section{Regularized Inversion}

Conceptually, singularity or near-singularity of $\mathrm{X}^{\mathrm{t}} \mathrm{QX}$ (as occurs when large numbers of parameters require estimation and/ or when the information content of the calibration dataset with respect to estimated parameters is poor) can be remedied through the addition of extra "observations" to the parameter estimation process which pertain directly to the parameters requiring estimation. For example, it may be "observed" that each parameter is equal to a certain, user-supplied value; presumably this value will have been chosen to be realistic in terms of the system property which the parameter represents. Alternatively (or as well), it may be "observed" that certain pairs of parameters are equal, or have values which observe a certain ratio or difference. 
Let these "regularization relationships" be represented by the operator $z$ acting on the parameter set $p$, and let the "observed" values of these relationships be represented by j. Then the regularization relationships (also referred to as "regularization constraints" herein) can be represented by the equation:

$$
z(p)=\mathrm{j}
$$

the linearized form of which is:

$$
\mathrm{Zp}=\mathrm{j}
$$

where $Z$ is the J acobian of the $z$ operator. Note that, as is discussed below, it is not essential that Equations $6 a$ and $6 \mathrm{~b}$ be exactly observed, only that they be observed to the maximum extent possible in calibrating the model.

If the regularization constraints are given sufficient weight in comparison with the observation weights encapsulated in Q, a well-posed inverse problem will have been formulated. Mathematically, this problem is then iteratively solved for the parameters $\mathrm{p}$ using the equation:

$$
p-p_{0}=\left(X^{t} Q X+\beta^{2} Z^{t} S Z+\lambda I\right)^{-1}\left(X^{t} Q\left[h-h_{0}\right]+\beta^{2} Z^{t} S\left[j-j_{0}\right]\right)
$$

In Equation 7, jo represents the right side of Equation 6a when current parameter values $\mathrm{p}_{0}$ are substituted for $\mathrm{p}$ in this equation. $\mathrm{S}$ is a "relative weight matrix" assigned to the regularization observations $\mathrm{j}$; it has the same role for regularization observations as Q does for field observations. All of the relative regularization weights encapsulated in $S$ are multiplied by a "regularization weight factor" $\beta^{2}$ in Equation 7 prior to calculation of p-po.

Selection of an appropriate value for $\beta^{2}$ is critical. If its value is too high the parameter estimation process will ignore the measurement dataset $h$ in favor of fitting the regularization observations $j$. If it is too small, the regularization observations will not endow the parameter estimation process with the numerical stability which it needs in order to obtain estimates for the parameters $\mathrm{p}$.

Equation 7 can be shown to constitute a constrained minimization problem in which a "regularization objective function" $\Phi_{\mathrm{r}}$ defined as: 


$$
\Phi_{\mathrm{r}}=[z(p)-j] \mathrm{S}[z(p)-j]
$$

is minimized subject to the constraint that $\Phi_{\mathrm{m}}$ of Equation 3 rises no higher than a user-specified value, referred to herein as the "target measurement objective function." Thus the user informs the regularized inversion process of the level of model-to-measurement misfit required; this process then enforces the regularization constraints defined through Equation $6 a$ to the maximum extent that it can by minimizing $\Phi_{\mathrm{r}}$ subject to the constraint that $\Phi_{\mathrm{m}}$ rises no higher than the target level. If the target measurement objective function cannot be achieved, the regularized inversion process simply minimizes $\Phi_{\mathrm{m}}$; however, where minimization of $\Phi_{\mathrm{m}}$ would otherwise be an unstable process due to parameter nonuniqueness, stability of this process is maintained by seeking that set of parameters lying within the elongate $\Phi_{\mathrm{m}}$ valley that also minimizes $\Phi_{\mathrm{r}}$. In either case, the regularization weight factor $\beta^{2}$ can be viewed as a Lagrange multiplier associated with the constrained minimization problem, and it is recalculated during every iteration of the regularized nonlinear parameter estimation process using a bisection algorithm based on local linearization of the constrained minimization problem about current parameter values.

Note the continued inclusion of the Marquardt lambda in Equation 7. Its value is adjusted as needed from iteration to iteration as a practical measure to enhance optimization efficiency and to ensure stability of the parameter estimation process should $\mathrm{X}^{\mathrm{t}} \mathrm{QX}+\beta^{2} \mathrm{Z}^{\mathrm{t}} \mathrm{SZ}$ become ill-conditioned through use of an inappropriately low value for $\beta^{2}$. This can occur where regularization constraints are poorly formulated, or where too good a fit is sought between model outputs and field measurements, requiring that regularization constraints be abandoned in pursuit of this fit. Often it occurs for a combination of these reasons, where weights on some regularization constraints must be lowered for attainment of a good fit between model outputs and field measurements, but where the relaxation of regularization constraints then leads to unestimability of those model parameters whose estimation is not realized through attainment of this fit.

Formulation of the inverse problem as a constrained minimization problem through use of Equation 7 allows many more parameters to be estimated than would otherwise be possible, thereby ensuring that maximum information is extracted from the calibration dataset. If the relationships of Equation 6 are realistic, the fact that estimated parameters are such as to ensure minimal deviation from these relationships heightens the prob- 
ability that estimated parameters will themselves be realistic. However, a practical problem that is often encountered when using the Tikhonov method is that the regularization weight matrix S must be supplied ahead of the regularized inversion process; furthermore, it is not adjusted through this process except for global multiplication by $\beta^{2}$. Ideally, individual regularization constraints described by the rows of Equation 6 should be more strongly enforced where the information content of the calibration dataset is insufficient to require their contravention for the sake of obtaining an appropriate level of model-to-measurement fit. However because it is almost impossible to know ahead of the calibration process the extent to which this should occur for each of the different relationships encapsulated in $z$, it is often very difficult to supply an S matrix that is an appropriate complement to the current calibration dataset.

\section{Adaptive Regularization}

An "adaptive regularization" methodology is now presented which overcomes this problem in many modeling contexts. The set of regularization constraints described by Equation 6 is subdivided into groups; if desired, each constraint can be assigned to its own group. The set of model parameters $\mathrm{p}$ is then supplemented by an additional parameter set $\mathrm{p}_{\mathrm{r}}$, with one new parameter being defined for each new regularization group. Each such parameter is, in fact, the inverse of a group-specific regularization weight multiplier; this group-specific weight multiplier is applied in addition to the global weight multiplier $\beta^{2}$ depicted in Equation 7, the latter being adjusted as part of the constrained minimization process as described above. Regularization constraints are then provided for the elements of $\mathrm{p}_{\mathrm{r}}$ so that these too can be estimated as part of the regularized inversion process. Each such constraint comprises the "observation" that the respective element of $\mathrm{p}_{\mathrm{r}}$ is zero.

The reformulated regularized inversion problem remains a constrained minimization process, and thus still seeks to find a parameter set that either minimizes the measurement objective function $\Phi_{\mathrm{m}}$, or reduces it to a user-specified target level, while ensuring that the regularization objective function $\Phi_{\mathrm{r}}$ is conditionally minimized. Because conditional minimization of the regularization objective function now requires maximization of weights assigned to individual or groups of regularization constraints, these weights are applied as strongly as possible, thereby maximizing the extent to which the corresponding regularization relationships encapsulated in Equation 6 are adhered. However, with the calculation of the over- 
all regularization weight factor $\beta^{2}$ by the constrained minimization process being such as to allow minimization of the target measurement objective function, or achievement of a user-specified target for this function, these regularization constraints are not so strongly enforced that model-tomeasurement fit is compromised. Thus, the regularized inversion process itself ensures that the strength of enforcement of regularization constraints on parameter values or relationships complements the information content of the calibration dataset in relation to these parameters. As a result, regularization constraints are automatically applied more strongly where the attainment of a satisfactory level of model-to-measurement fit does not require otherwise, thus overcoming a disadvantage of the Tikhonov method. The outcome is a numerically stable regularized inversion process that achieves a desired level of model-to-measurement fit with impressive run economy, and that yields sensible values for model parameters.

Like all numerical strategies, this adaptive regularization methodology is more suitable for use in some contexts than in others. It is certainly not the only means by which numerical stability of a regularized inversion process can be achieved, for so-called "subspace methods" (Aster et al. 2005) are very effective in this regard. However, use of the present methodology can be beneficial in those modeling contexts where the means by which numerical stability is achieved is just as important as the achievement of that stability itself. In general, where the necessity for parameters to observe key values or relationships to the maximum extent possible without compromising fit between model outputs and field measurements is a critical part of the calibration process, then the adaptive regularization methodology described herein will serve that calibration process well; such a case is demonstrated in the following section. However, the need to introduce extra parameters into the calibration process in order to guarantee enforcement of desired parameter relationships does place some restrictions on the method. Where such relationships fall into a relatively small number of distinct groups, and/ or where the number of parameters requiring estimation is not such as to introduce vastly different levels of "estimability" between them (thus requiring the introduction of many new parameters in order to accommodate the differential strengths with which regularization constraints must be applied), the above method has proven very successful. However, where large numbers of parameters require estimation, and where differences in estimability between them are likely to cover a broad range, recourse to subspace methods becomes a necessity. 
Unfortunately, in this case, the guarantee of numerical stability that accompanies use of such methods is attained at the cost of loss of ability on the part of the modeler to insist on the observance of specified parameter relationships in attaining that stability. 


\section{Examples}

Use of the methodologies discussed in the preceding section are now demonstrated by applying them to the calibration of two different HEC-HMS models deployed to the Goodwin Creek Experimental Watershed. Goodwin Creek is an 8.26-square-mile experimental watershed (upland erosion, instream sediment transport, and watershed hydrology) operated by the Agricultural Research Service (ARS) of the United States Department of Agriculture (USDA). The Goodwin Creek watershed is located in northern Mississippi, approximately 60 miles south of Memphis, TN. Goodwin Creek is divided into 14 nested subwatersheds with a flow measuring flume constructed at each of the subwatershed outlets. The drainage areas above the gauging sites range from 0.63 to 8.26 square miles. Thirty-one standard recording rain gauges are uniformly located within and just outside of the watershed. For further details about the Goodwin Creek Experimental Watershed, see Blackmarr (1995). Digital elevation modelderived subwatershed boundaries and stream network, and rain and stream gauge locations are shown in Figure 1.

The first HEC-HMS model was applied to the 39.8-acre drainage area above streamflow gauging station number 9 in Goodwin Creek. Precipitation data from two nearby gauges and evaporation data associated with four surrounding locations were supplied as meteorological forcing data to support HEC-HMS continuous hydrologic simulation using the deficit constant loss method; for details about the deficit constant loss method within HEC-HMS, see HEC (2005). 


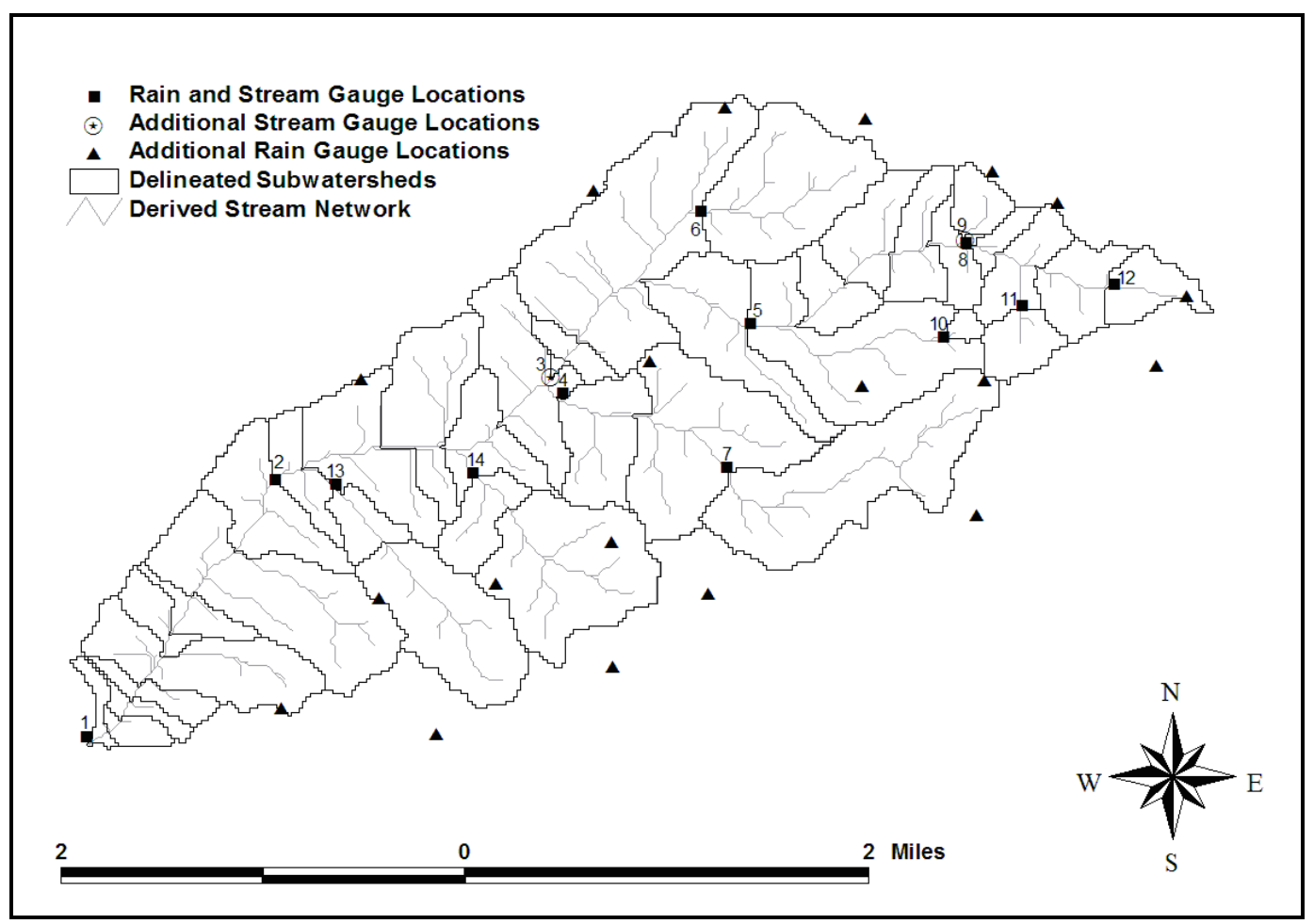

Figure 1. Goodwin Creek Experimental Watershed, delineated subwatersheds, derived stream network, rain and numbered streamflow gauge locations.

Estimation of six HEC-HMS parameters was undertaken by matching observed and simulated flow data over 17 non-contiguous time intervals spanning the period 1J an 1989 to 29 Feb 1992. The 17 periods were identified based on a manual inspection of the observed flow data. The calibration period 1J an 1989 to 29 Feb 1992 was selected based on the determination that there was no land use/ land cover alteration within the subwatershed for the period 1987 through 1992. Two calibration experiments were performed with the first HEC-HMS model. The first calibration experiment involved comparing only simulated and observed flows; the second calibration experiment involved comparing simulated and observed flows and base flows. Over the 17 non-contiguous time intervals spanning the calibration period 1J an 1989 to 29 Feb 1992, the first experiment involved matching simulated flows with observed flows above a predetermined threshold (which was uniquely determined for each of the 17 time intervals based on a manual inspection of the data); whereas, the second experiment involved matching not only simulated flows with observed flows (above the predetermined threshold), but also matching simulated base flows with observed base flows for the same times simulated and observed flows were matched. Hence, the first experiment resulted in a total of 2,053 flow observations for use in the calibration proc- 
ess; whereas, the second experiment resulting in a total of 4,106 observations for use in the calibration process.

In each case, the objective function was defined as the sum of weighted squared differences between modeled and observed log-transformed flow data, with all weights assigned a value of 1.0. Thus $\mathbf{h}$ of Equation 3 was comprised of the logs of flow data, while the model represented by $\mathbf{X}$ in these equations calculated the model-generated counterparts to these logged flow data. $\mathbf{Q}$ was the identity matrix. For both experiments with the first HEC-HMS model, the simulation time interval was one hour, which equaled the temporal resolution of the input precipitation data.

Table 2 lists the 17 non-contiguous time intervals and the specified thresholds. Figure 2 is a plot of the observed flow data that were compared with simulated flows to calibrate, for both experiments, the first HEC-HMS model. The "observed" base flow data for comparing with HEC-HMS simulated base flow, for the second experiment with the first model, was determined using a quickflow digital filter (Nathan and McMahon 1990), with a value of 0.999 specified for the scaling parameter. For purposes of illustration, base flow "observations," together with the "observed" quickflow and the total observed flow, for a specific period during the first of the 17 time intervals, are plotted in Figure 3. Examining Figure 3, clearly the perceptual model (Beven 2001) was formulated to be consistent with past research (Downer and Ogden 2003) in that the watershed system is dominated by quickflow response.

Table 2. Non-contiguous time intervals and thresholds, both which determined model to measurement misfit for both experiments with the first model.

\begin{tabular}{|l|l|l|}
\hline Number & Time Interval & Threshold (cfs) \\
\hline 1 & $01 / 02 / 1989$ 00:00:00 - 02/13/1989 23:00:00 & 0.1 \\
\hline 2 & $02 / 18 / 1989$ 06:00:00 - 02/21/1989 12:00:00 & 0.1 \\
\hline 3 & $02 / 27 / 1989$ 02:00:00 - 05/17/1989 23:00:00 & 0.1 \\
\hline 4 & $05 / 22 / 1989$ 00:00:00 - 08/08/1989 23:00:00 & 0.1 \\
\hline 5 & $01 / 03 / 1990$ 18:00:00 - 01/08/1990 08:00:00 & 0.1 \\
\hline 6 & $01 / 28 / 1990$ 20:00:00 - 01/29/1990 23:00:00 & 0.1 \\
\hline 7 & $02 / 09 / 1990$ 13:00:00 - 03/13/1990 23:00:00 & 0.1 \\
\hline 8 & $04 / 06 / 1990$ 00:00:00 - 04/06/1990 23:00:00 & 0.1 \\
\hline 9 & $04 / 21 / 1990$ 00:00:00 - 06/04/1990 23:00:00 & 0.05 \\
\hline
\end{tabular}




\begin{tabular}{|l|l|l|}
\hline 10 & $07 / 22 / 1990$ 21:00:00 - 07/23/1990 06:00:00 & 0.05 \\
\hline 11 & $07 / 31 / 1990$ 13:00:00 - 07/31/1990 18:00:00 & 0.05 \\
\hline 12 & $02 / 05 / 1991$ 03:00:00 - 02/06/1991 23:00:00 & 0.2 \\
\hline 13 & $02 / 18 / 1991$ 01:00:00 - 03/22/1991 23:00:00 & 0.1 \\
\hline 14 & $03 / 28 / 1991$ 13:00:00 - 05/15/1991 23:00:00 & 0.1 \\
\hline 15 & $06 / 22 / 1991$ 07:00:00 - 09/30/1991 00:00:00 & 0.05 \\
\hline 16 & $01 / 08 / 1992$ 06:00:00 - 01/16/1992 23:00:00 & 0.1 \\
\hline 17 & $02 / 14 / 1992$ 10:00:00 - 02/16/1992 00:00:00 & 0.1 \\
\hline
\end{tabular}

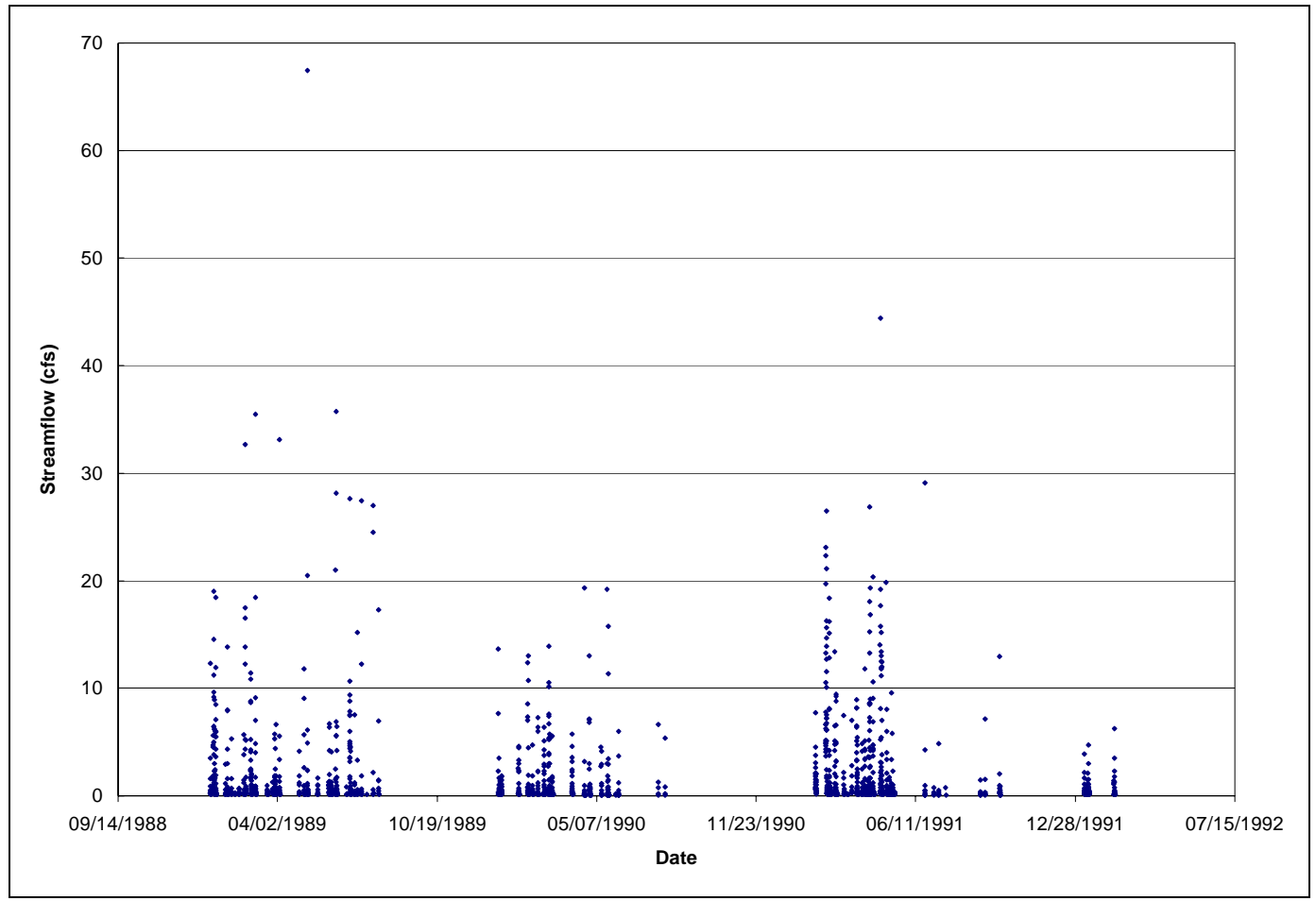

Figure 2. Plot of observed flow data used to calibrate the first HEC-HMS model. 


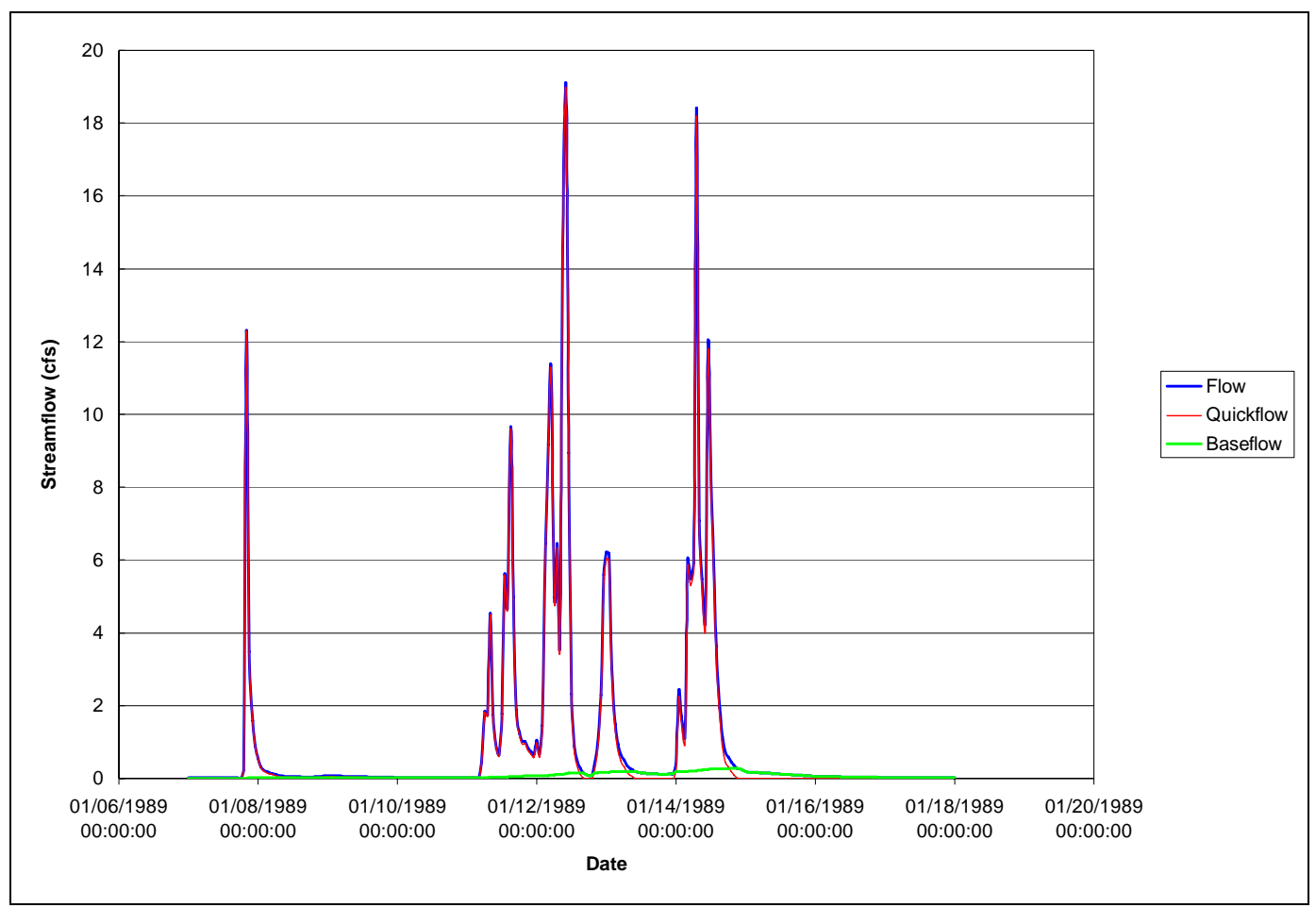

Figure 3. Observed flow, quickflow, and base flow data at gauge 9 in Goodwin Creek Experimental Watershed.

Table 3 lists the names and functions of the HEC-HMS parameters estimated through the calibration process. Also shown in this table are the bounds applied to these parameters; guidance in the setting of these bounds was obtained from HEC (2000). In order to ensure estimation of physically acceptable values for the initial deficit (ID) and maximum storage (MS) parameters in the deficit constant loss model, the two adjustable model parameters LENGTH and A were related to the initial deficit and maximum storage in the following manner

$$
\begin{gathered}
\text { ID = LENGTH } \cdot \text { A } \\
\text { MS }=\text { LENGTH } \cdot \text { porosity }
\end{gathered}
$$

As noted in Table 3, the adjustable model parameter A was specified to be less than the porosity of the soil, which was fixed to be 0.36 . In order to better accommodate scaling issues resulting from the use of different units for different parameters, and in an attempt to decrease the degree of nonlinearity of the parameter estimation problem, the logs of these parameters were estimated instead of their native values; past experience has demonstrated that greater efficiency and stability of the parameter estima- 
tion process can often be achieved through this means (Doherty and Skahill 2006).

Table 3. HEC-HMS parameters, their functions, and constraints imposed during the calibration process.

\begin{tabular}{|l|l|l|}
\hline Parameter Name & Parameter Function & $\begin{array}{l}\text { Bounds Imposed During } \\
\text { Calibration }\end{array}$ \\
\hline LENGTH & Depth of the active soil layer & $0.003-54.681 \mathrm{in}$. \\
\hline A & LENGTH · A = initial deficit; A < porosity & $0.001-0.359998$ \\
\hline CONSTLOSS & $\begin{array}{l}\text { Infiltration rate when the soil layer is satu- } \\
\text { rated }\end{array}$ & $0.001-11.810 \mathrm{in} . / \mathrm{hr}$ \\
\hline SNYDERTP & Snyder unit hydrograph standard lag & $0.100-500 \mathrm{hr}$ \\
\hline SNYDERCP & $\begin{array}{l}\text { Snyder unit hydrograph peaking coeffi- } \\
\text { cient }\end{array}$ & $0.100-1$ \\
\hline GWSTOCOEFF & $\begin{array}{l}\text { Groundwater storage coefficient for the } \\
\text { linear reservoir }\end{array}$ & $0.100-10000 \mathrm{hr}$ \\
\hline
\end{tabular}

The second HEC-HMS model was applied to the drainage areas above streamflow gauging station numbers 8, 9, 11, and 12 in Goodwin Creek. The second HEC-HMS model included separate submodels for the drainage areas upstream of the four noted streamflow gauging stations (station numbers 8, 9, 11, and 12) located within the watershed. Precipitation data from seven nearby gauges and evaporation data associated with four surrounding locations were supplied as meteorological forcing data to support HEC-HMS continuous hydrologic simulation using the deficit constant loss method.

The names and roles of model parameters selected for adjustment through the calibration process are provided in Table 3. Also listed are the bounds on these parameters imposed during the parameter estimation process, these being set in accordance with available guidance HEC (2000). Four instances of all the parameters listed in Table 3 required estimation, one instance for each subwatershed model. Thus a total of 24 model parameters required estimation through the calibration process. As with the first HEC-HMS model, in order to ensure estimation of physically acceptable values for the initial deficit and maximum storage parameters in the deficit constant loss model, the adjustable model parameters LENGTH and A, for each subwatershed, were related to the initial deficit and maximum storage as specified in Equations 9 and 10, respectively. And as noted in 
Table 3, the adjustable model parameter A, for each subwatershed, was specified to be less than the porosity of the soil, which was fixed to be 0.36. Also as with the first HEC-HMS model, in order to better accommodate scaling issues resulting from the use of different units for different parameters, and in an attempt to decrease the degree of nonlinearity of the parameter estimation problem, the logs of these parameters were estimated instead of their native values as past experience has demonstrated that greater efficiency and stability of the parameter estimation process can often be achieved through this means (Doherty and Skahill 2006).

Simultaneous estimation of the parameters listed in Table 3 for the four different subwatersheds allows an important piece of information to be included in the parameter estimation process. Namely that, due to their geographical proximity and similarity of land use, soil type, and other geomorphic and anthropogenic conditions, parameter values employed in the different subwatershed models are not expected to be significantly different. To accommodate this condition, a series of regularization constraints effecting an assumed similarity condition across the subwatersheds was included in the regularized parameter estimation process. That is, respective log differences of identical parameter types between subwatersheds were ascribed an "observed value" of zero. The advantage of supplying such information through regularization constraints rather than through "hardwired" parameter equality is that the regularized inversion process has the option of introducing parameter differences if this is a requirement for obtaining a good fit between modeled and observed flows at each of the streamflow gauging stations. However, the constrained optimization algorithm which underpins the regularized inversion process guarantees that only the minimum amount of inter-parameter variability required to achieve this level of fit is introduced. Thus, subwatershed individuality is recognized at the same time as subwatershed similarity.

Estimation of the 24 adjustable parameters for the second HEC-HMS model was undertaken by matching observed and simulated flows over 23 , 11, 19, and 18 non-contiguous time intervals spanning the period $1 \mathrm{~J}$ an 1988 to 31 Dec 1990 for stations 8, 9, 11, and 12, respectively. The noted periods were identified based on a manual inspection of the observed flow data. The calibration period 1J an 1988 to 31 Dec 1990 was selected based on the determination that there was no land/ use land cover alteration within the four subwatersheds for the period 1988 through 1990. Over the $23,11,19$, and 18 non-contiguous time intervals spanning the calibration 
period 1J an 1988 to 31 Dec 1990 for stations 8, 9, 11, and 12, respectively, model calibration involved matching simulated flows with observed flows above a predetermined threshold (which was uniquely determined for each of the 23, 11, 19, and 18 non-contiguous time intervals for stations 8 , 9,11 , and 12 , respectively, based on a manual inspection of the data). This resulted in a total of 8,480 flow observations for use in the calibration process of the second HEC-HMS model.

As with the first HEC-HMS model, the objective function for the second HEC-HMS model was defined as the sum of weighted squared differences between modeled and observed log-transformed flows, with all weights assigned a value of 1.0. Thus $\mathbf{h}$ of Equation 3 was comprised of the logs of flows, while the model represented by $\mathbf{X}$ in these equations calculated the model-generated counterparts to these logged flows. $\mathbf{Q}$ was the identity matrix. For the second HEC-HMS model, the simulation time interval was one hour, which equaled the temporal resolution of the input precipitation data.

Tables 4 to 7 list the 23, 11, 19, and 18 non-contiguous time intervals for stations $8,9,11$, and 12, respectively, and their respective thresholds. Figures 4 to 7 are plots of the observed flow data that were compared with simulated flows to calibrate the second HEC-HMS model.

Table 4. For the second HEC-HMS model, non-contiguous time intervals and thresholds associated with station 8 .

\begin{tabular}{|l|l|l|}
\hline Number & Time Interval & Threshold (cfs) \\
\hline 1 & $01 / 18 / 1988$ 20:00:00 - 01/20/1988 00:00:00 & 0.1 \\
\hline 2 & $02 / 02 / 1988$ 02:00:00 - 02/05/1988 00:00:00 & 0.1 \\
\hline 3 & $02 / 14 / 1988$ 16:00:00 - 03/14/1988 00:00:00 & 0.2 \\
\hline 4 & $04 / 06 / 1988$ 00:00:00 - 04/07/1988 00:00:00 & 0.1 \\
\hline 5 & $11 / 19 / 1988$ 20:00:00 - 11/27/1988 00:00:00 & 0.2 \\
\hline 6 & $12 / 21 / 1988$ 00:00:00 - 01/02/1989 00:00:00 & 0.2 \\
\hline 7 & $01 / 07 / 1989$ 16:00:00 - 02/04/1989 10:00:00 & 0.1 \\
\hline 8 & $02 / 12 / 1989$ 18:00:00 - 03/08/1989 00:00:00 & 0.1 \\
\hline 9 & $03 / 29 / 1989$ 04:00:00 - 04/06/1989 00:00:00 & 0.1 \\
\hline 10 & $05 / 05 / 1989$ 02:00:00 - 05/23/1989 00:00:00 & 0.3 \\
\hline 11 & $06 / 08 / 1989$ 02:00:00 - 06/17/1989 00:00:00 & 0.2 \\
\hline 12 & $07 / 01 / 198906: 00: 00-07 / 17 / 1989$ 00:00:00 & 0.2 \\
\hline 13 & $07 / 30 / 1989$ 20:00:00 - 08/07/1989 16:00:00 & 0.1 \\
\hline 14 & $11 / 06 / 1989$ 00:00:00 - 11/09/1989 00:00:00 & 0.2 \\
\hline
\end{tabular}




\begin{tabular}{|l|l|l|}
\hline 15 & $12 / 30 / 1989$ 02:00:00 - 01/08/1990 12:00:00 & 0.2 \\
\hline 16 & $01 / 17 / 1990$ 14:00:00 - 01/30/1990 00:00:00 & 0.3 \\
\hline 17 & $02 / 02 / 1990$ 00:00:00 - 03/18/1990 00:00:00 & 0.2 \\
\hline 18 & $03 / 28 / 1990$ 12:00:00 - 04/08/1990 00:00:00 & 0.2 \\
\hline 19 & $04 / 21 / 1990$ 08:00:00 - 05/22/1990 12:00:00 & 0.3 \\
\hline 20 & $06 / 03 / 1990$ 00:00:00 - 06/04/1990 00:00:00 & 0.2 \\
\hline 21 & $11 / 28 / 199000: 00: 00-12 / 04 / 199000: 00: 00$ & 0.2 \\
\hline 22 & $12 / 17 / 199000: 00: 00-12 / 23 / 199000: 00: 00$ & 0.2 \\
\hline 23 & $12 / 27 / 199004: 00: 00-12 / 31 / 1990$ 23:00:00 & 0.2 \\
\hline
\end{tabular}

Table 5. For the second HEC-HMS model, non-contiguous time intervals and thresholds associated with station 9 .

\begin{tabular}{|l|l|l|}
\hline Number & Time Interval & Threshold (cfs) \\
\hline 1 & $01 / 02 / 1989$ 00:00:00 - 02/13/1989 23:00:00 & 0.1 \\
\hline 2 & $02 / 18 / 1989$ 06:00:00 - 02/21/1989 12:00:00 & 0.1 \\
\hline 3 & $02 / 27 / 1989$ 02:00:00 - 05/17/1989 23:00:00 & 0.1 \\
\hline 4 & $05 / 22 / 1989$ 00:00:00 - 08/08/1989 23:00:00 & 0.1 \\
\hline 5 & $01 / 03 / 1990$ 18:00:00 - 01/08/1990 08:00:00 & 0.1 \\
\hline 6 & $01 / 28 / 1990$ 20:00:00 - 01/29/1990 23:00:00 & 0.1 \\
\hline 7 & $02 / 09 / 1990$ 13:00:00 - 03/13/1990 23:00:00 & 0.1 \\
\hline 8 & $04 / 06 / 1990$ 00:00:00 - 04/06/1990 23:00:00 & 0.1 \\
\hline 9 & $04 / 21 / 1990$ 00:00:00 - 06/04/1990 23:00:00 & 0.05 \\
\hline 10 & $07 / 22 / 1990$ 21:00:00 - 07/23/1990 06:00:00 & 0.05 \\
\hline 11 & $07 / 31 / 1990$ 13:00:00 - 07/31/1990 18:00:00 & 0.05 \\
\hline
\end{tabular}

Table 6. For the second HEC-HMS model, non-contiguous time intervals and thresholds associated with station 11.

\begin{tabular}{|l|l|l|}
\hline Number & Time Interval & Threshold (cfs) \\
\hline 1 & $01 / 18 / 1988$ 20:00:00 - 01/21/1988 00:00:00 & 0.1 \\
\hline 2 & $02 / 02 / 1988$ 02:00:00 - 03/14/1988 00:00:00 & 0.1 \\
\hline 3 & $04 / 02 / 1988$ 00:00:00 - 04/13/1988 00:00:00 & 0.1 \\
\hline 4 & $11 / 26 / 1988$ 02:00:00 - 02/04/1989 00:00:00 & 0.3 \\
\hline 5 & $02 / 12 / 1989$ 18:00:00 - 03/06/1989 10:00:00 & 0.2 \\
\hline 6 & $03 / 29 / 1989$ 04:00:00 - 04/05/1989 12:00:00 & 0.1 \\
\hline 7 & $05 / 05 / 1989$ 02:00:00 - 05/11/1989 00:00:00 & 0.1 \\
\hline 8 & $06 / 05 / 1989$ 16:00:00 - 06/17/1989 00:00:00 & 0.1 \\
\hline 9 & $07 / 01 / 198904: 00: 00-07 / 17 / 198900: 00: 00$ & 0.1 \\
\hline
\end{tabular}




\begin{tabular}{|l|l|l|}
\hline 10 & $07 / 30 / 1989$ 20:00:00 - 08/08/1989 00:00:00 & 0.1 \\
\hline 11 & $11 / 06 / 1989$ 00:00:00 - 11/09/1989 00:00:00 & 0.1 \\
\hline 12 & $12 / 30 / 1989$ 00:00:00 - 01/08/1990 10:00:00 & 0.2 \\
\hline 13 & $01 / 28 / 1990$ 18:00:00 - 03/03/1990 10:00:00 & 0.2 \\
\hline 14 & $03 / 07 / 1990$ 12:00:00 - 03/17/1990 00:00:00 & 0.3 \\
\hline 15 & $03 / 28 / 1990$ 10:00:00 - 04/07/1990 12:00:00 & 0.2 \\
\hline 16 & $04 / 21 / 1990$ 06:00:00 - 05/22/1990 10:00:00 & 0.2 \\
\hline 17 & $06 / 03 / 1990$ 02:00:00 - 06/04/1990 12:00:00 & 0.1 \\
\hline 18 & $11 / 27 / 1990$ 22:00:00 - 12/04/1990 00:00:00 & 0.1 \\
\hline 19 & $12 / 17 / 1990$ 00:00:00 - 12/31/1990 23:00:00 & 0.3 \\
\hline
\end{tabular}

Table 7. For the second HEC-HMS model, non-contiguous time intervals and thresholds associated with station 12 .

\begin{tabular}{|c|c|c|}
\hline Number & Time Interval & Threshold (cfs) \\
\hline 1 & 01/17/1988 00:00:00 - 01/20/1988 12:00:00 & 0.2 \\
\hline 2 & 02/02/1988 00:00:00 - 02/05/1988 00:00:00 & 0.1 \\
\hline 3 & 02/14/1988 18:00:00 - 04/12/1988 12:00:00 & 0.1 \\
\hline 4 & 11/19/1988 20:00:00 - 11/27/1988 12:00:00 & 0.1 \\
\hline 5 & 12/21/1988 00:00:00 - 12/31/1988 23:00:00 & 0.1 \\
\hline 6 & 01/11/1989 01:00:00 - 02/28/1989 23:00:00 & 0.1 \\
\hline 7 & 05/05/1989 01:00:00 - 05/10/1989 12:00:00 & 0.2 \\
\hline 8 & 06/04/1989 06:00:00 - 06/17/1989 00:00:00 & 0.1 \\
\hline 9 & 07/01/1989 04:00:00 - 07/17/1989 12:00:00 & 0.1 \\
\hline 10 & 08/06/1989 16:00:00 - 08/08/1989 00:00:00 & 0.1 \\
\hline 11 & 11/06/1989 00:00:00 - 11/09/1989 00:00:00 & 0.1 \\
\hline 12 & 12/30/1989 00:00:00 - 01/08/1990 12:00:00 & 0.2 \\
\hline 13 & 01/28/1990 18:00:00 - 02/04/1990 00:00:00 & 0.4 \\
\hline 14 & 02/09/1990 12:00:00 - 03/17/1990 00:00:00 & 0.3 \\
\hline 15 & 03/28/1990 08:00:00 - 04/07/1990 00:00:00 & 0.3 \\
\hline 16 & 04/21/1990 08:00:00 - 06/10/1990 00:00:00 & 0.2 \\
\hline 17 & 11/27/1990 22:00:00 - 11/28/1990 12:00:00 & 0.1 \\
\hline 18 & 12/17/1990 02:00:00 - 12/31/1990 23:00:00 & 0.2 \\
\hline
\end{tabular}




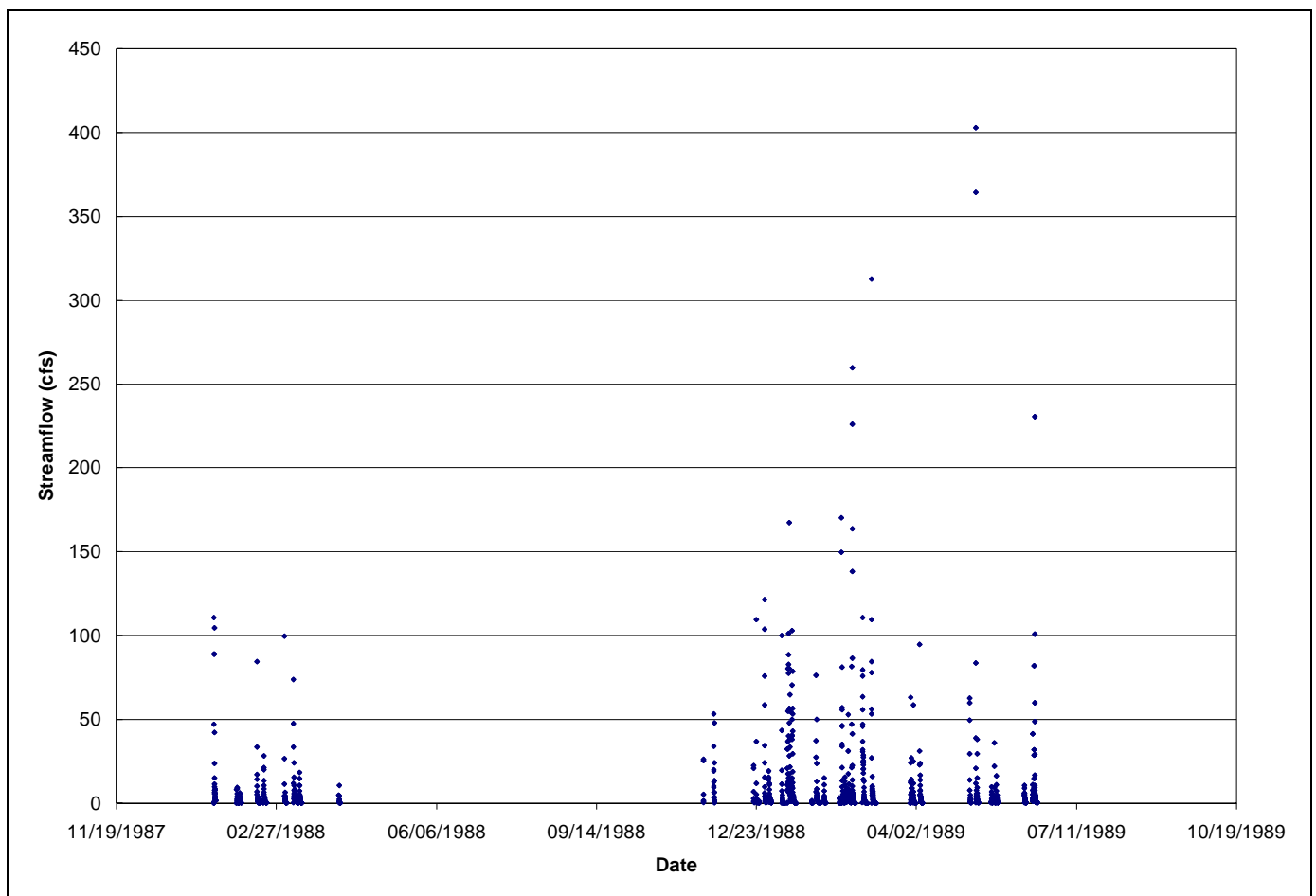

Figure 4. Plot of station 8 observed flow data used to calibrate the second HMS model.

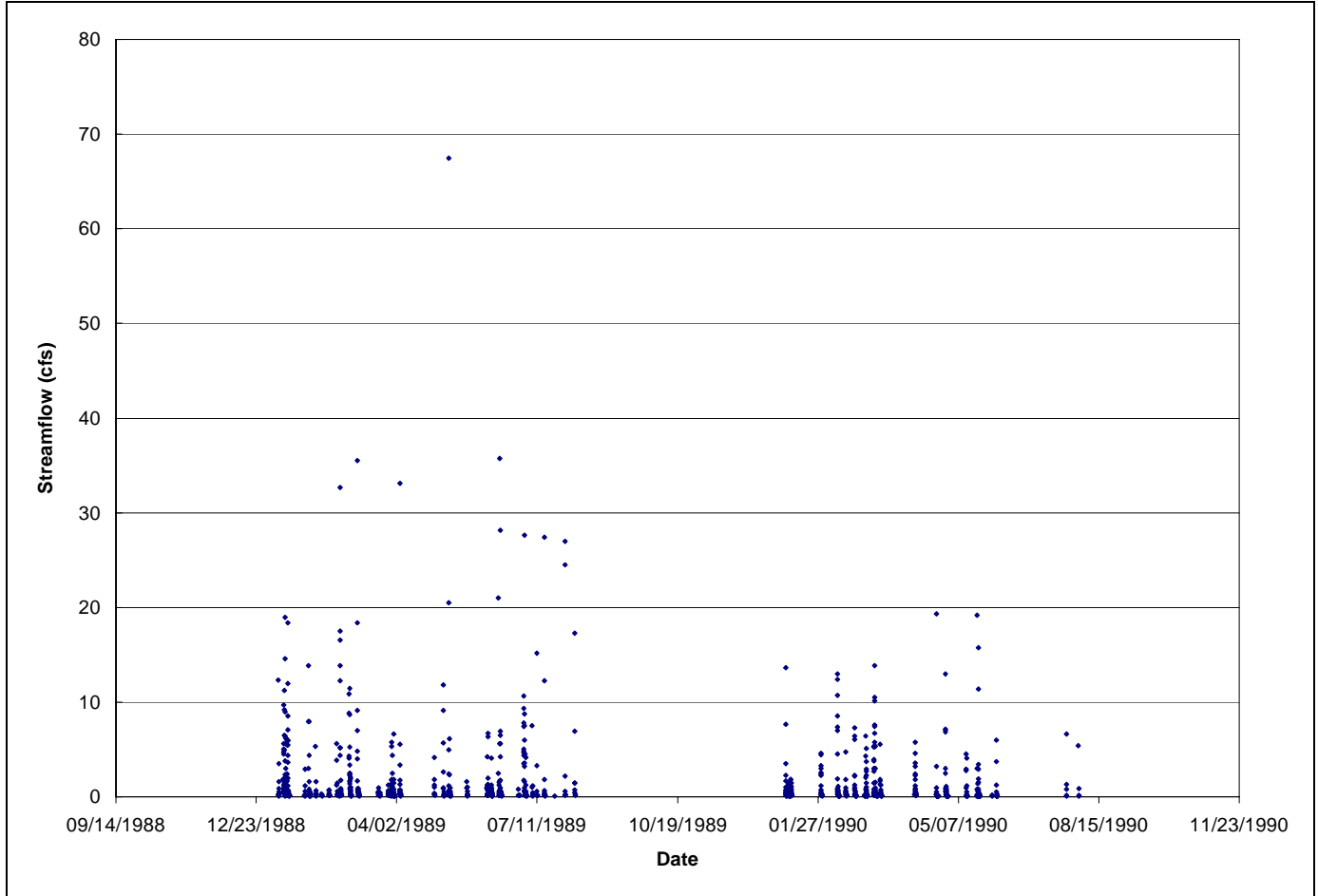

Figure 5. Plot of station 9 observed flow data used to calibrate the second HMS model. 


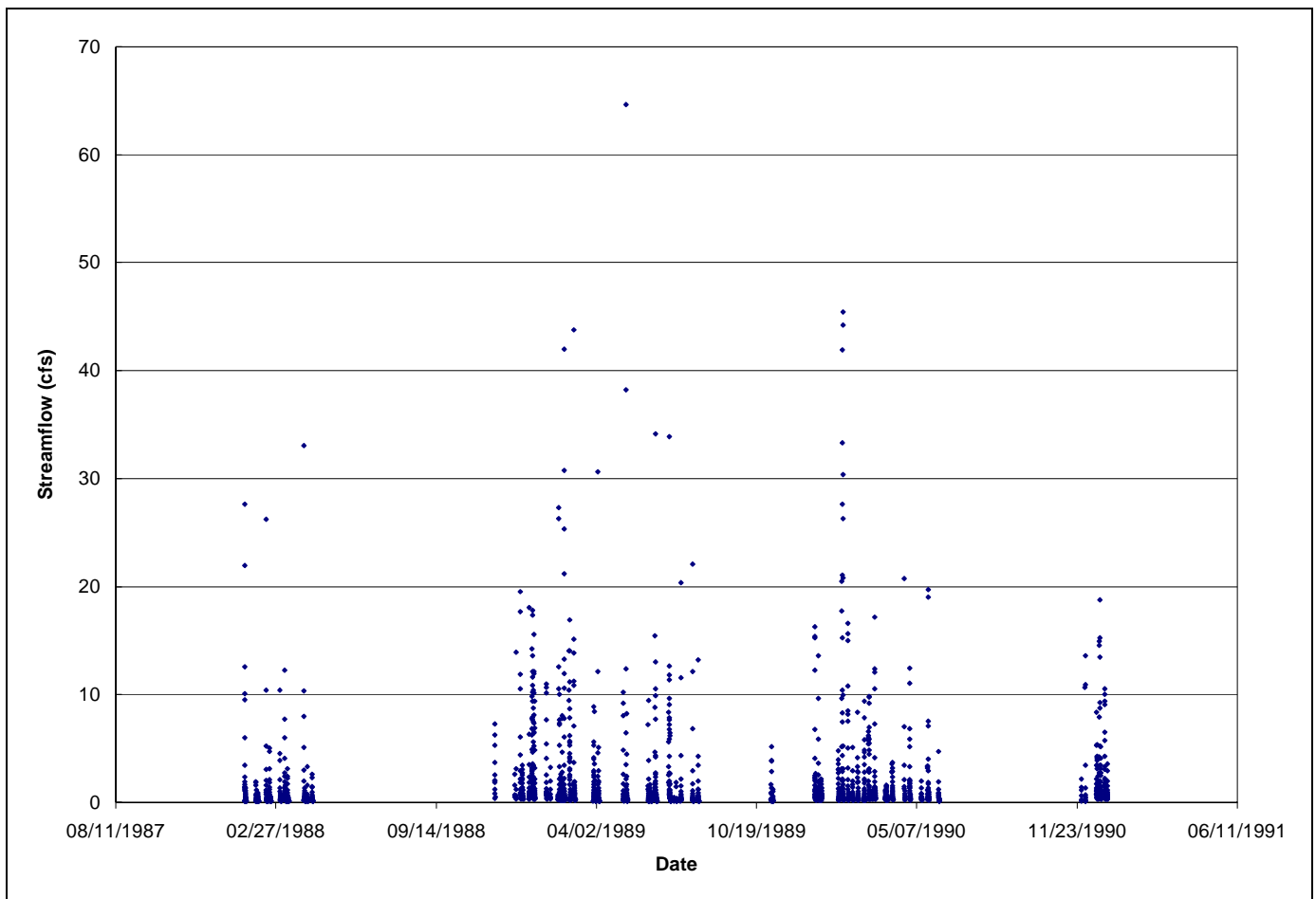

Figure 6. Plot of station 11 observed flow data used to calibrate the second HMS model.

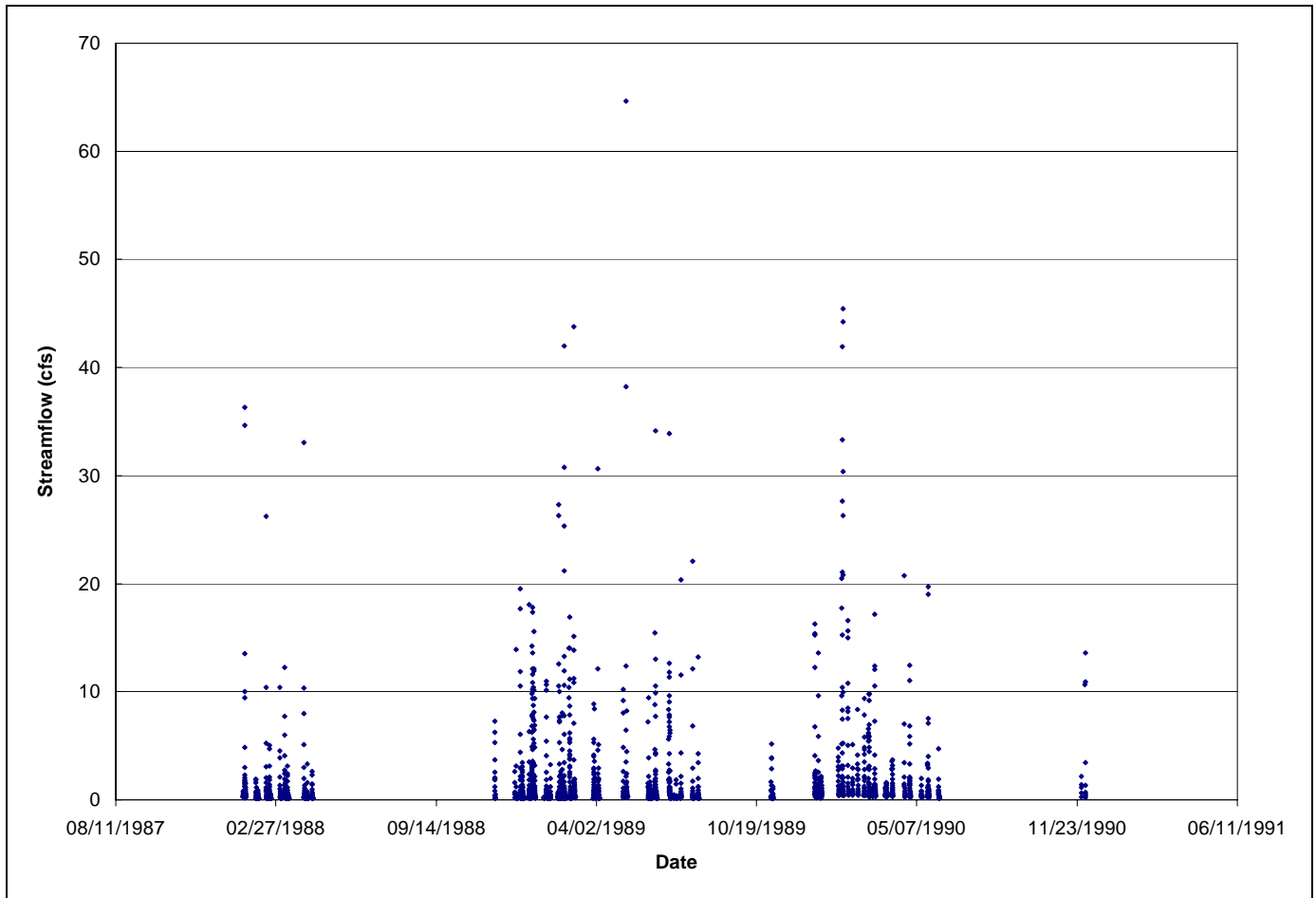

Figure 7. Plot of station 12 observed flow data used to calibrate the second HMS model. 


\section{Results}

\section{First HEC-HMS Model - Calibration Experiment 1}

In an attempt to locate as many local minima as possible, PD_MS2 was asked to run 100 inversion runs from a succession of starting values that were maximally distant from all previous parameter trajectories, as discussed above. These starting values were selected from 1,296 random parameter samples for which objective functions were calculated prior to the undertaking of any inversion runs.

Figure 8 depicts the outcomes of this exercise. Each of the six graphs appearing in this figure pertains to one of the six estimated parameters. For each graph the objective function is plotted on the $\mathrm{x}$ axis, while an optimized parameter value is plotted on the y axis. In each graph, each point represents the outcome of one PD_MS2-supervised inversion run; corresponding points from different graphs (representing corresponding values for different parameters) can be matched vertically through their common objective function. It is readily apparent from this figure that many of the outcomes of successive optimization runs are grouped into "parameter clumps" of nearly constant objective function value; these clumps define regions of attraction in parameter space. "Tight" clumps indicate a welldefined region of attraction; vertical spreading of clumps indicates difficulties in parameter identification through parameter correlation and/or insensitivity. For those minima situated at the bottom of broad objective function valleys defining different regions of attraction in parameter space, local minima are often in close proximity. Other local minima appear to exist in isolation from these more populous clumps.

For this calibration problem, the objective function has a value of 223.6 at its global minimum. Table 8 summarizes computed Nash-Sutcliffe efficiency scores (which are based on a comparison of the flow observations used in the calibration process) for each of the individual 17 noncontiguous time intervals. Figure 9 shows the fit between modeled and observed flows at this minimum for the flows that were compared for the seventh non-contiguous time interval listed in Table 2. 

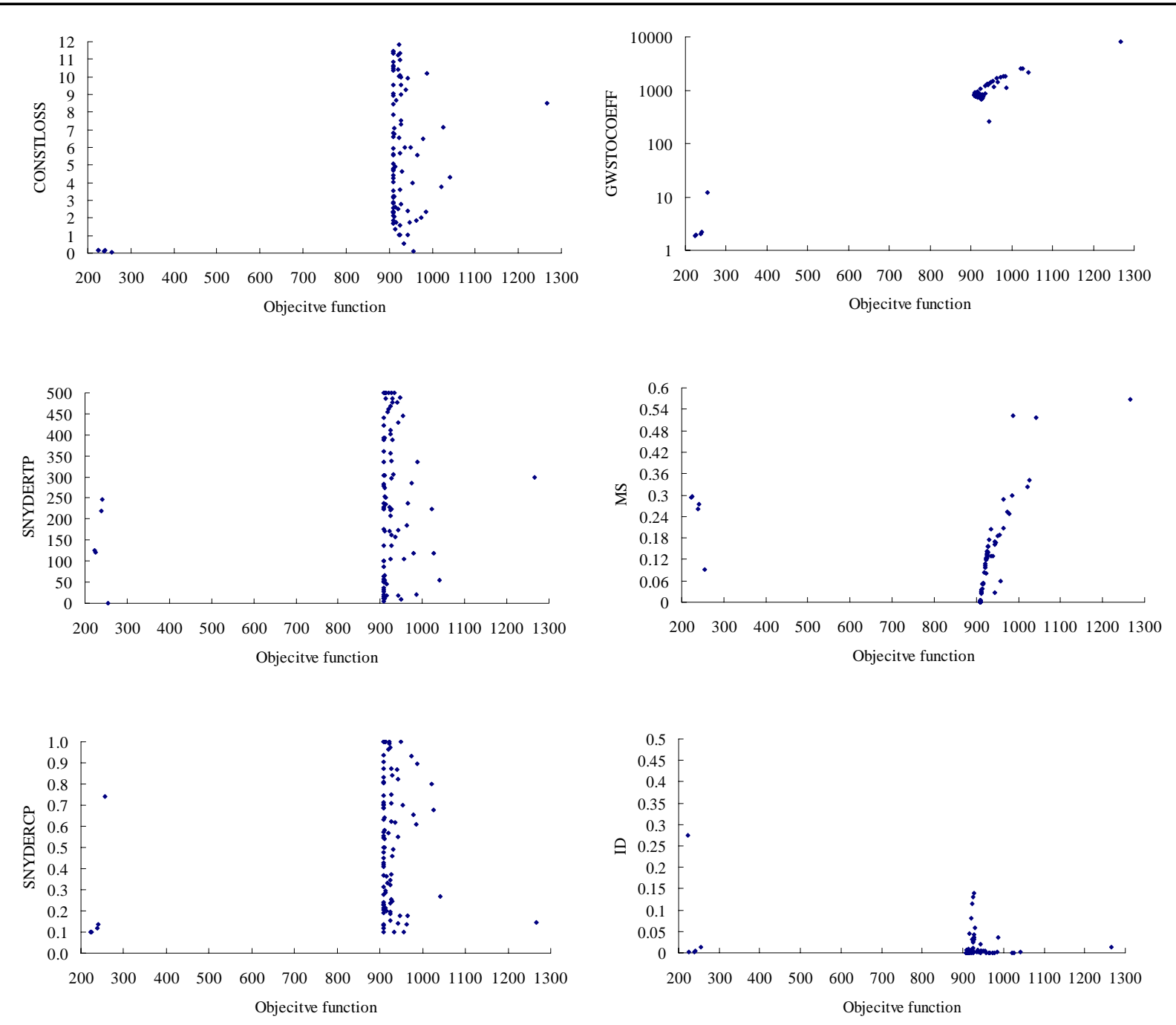

Figure 8. End-points in parameter space of 100 inversion runs undertaken under the control of PD_MS2. Parameters comprising an optimized set are linked vertically between graphs by objective function. TPI functionality was not operative.

Ninety-five percent confidence intervals for the parameters corresponding to the global objective function minimum are provided in Table 9, and these could only be calculated after fixing the parameter A, for A could not be defined at the global objective function minimum. The insensitivity of parameter A is exhibited upon inspection of Figure 8, wherein it is apparent that the global objective function minimum of 223.6 occurs in an elongate valley rather than a bowl (see the dotty plot for the initial deficit parameter, ID). 


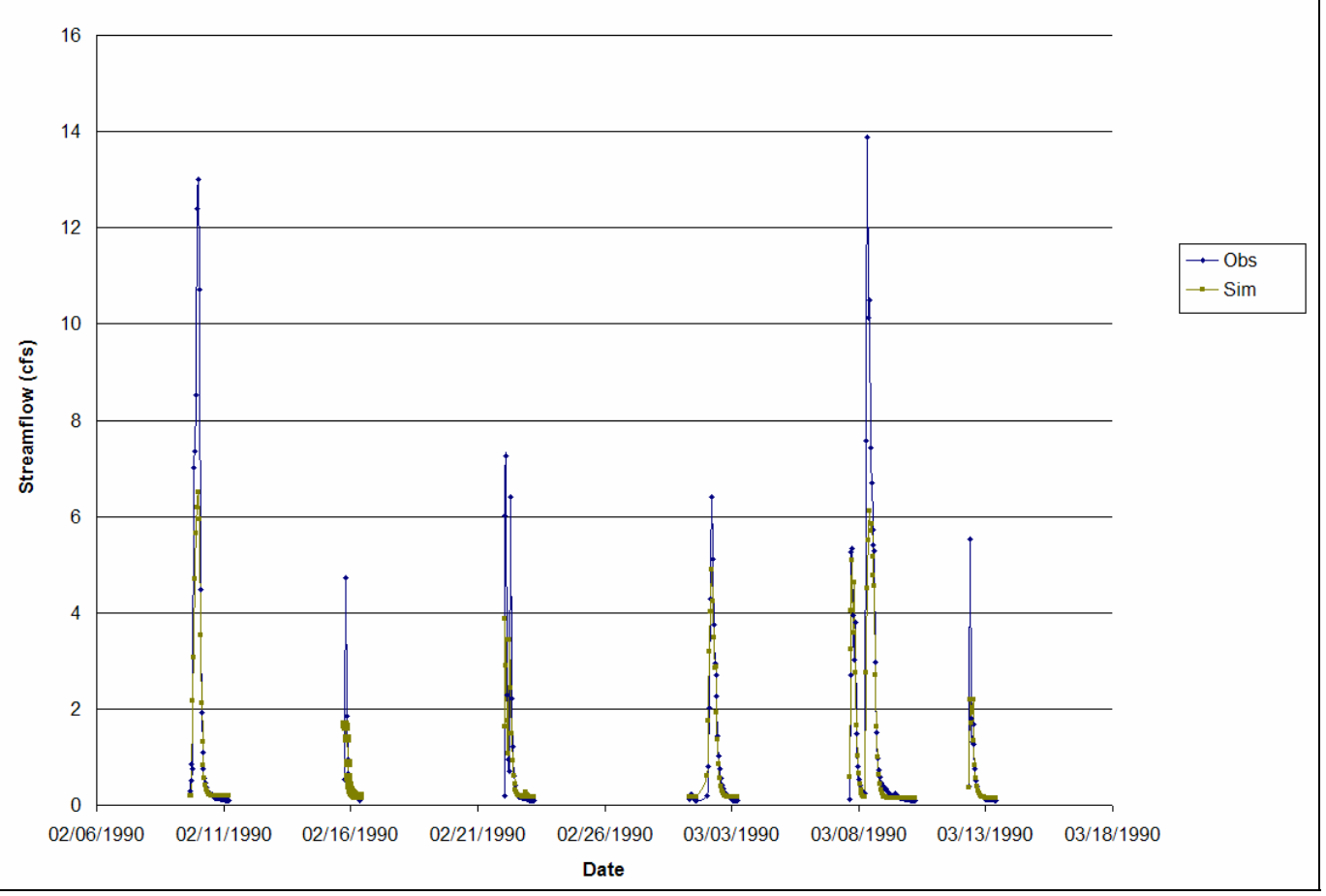

Figure 9. Simulated and observed flows at identified global minimum for the flows that were compared for the seventh non-contiguous time interval listed in Table 2.

Table 8. Nash-Sutcliffe efficiency associated with identified global minimum.

\begin{tabular}{|c|c|c|}
\hline Number & Number of Series Terms in this Interval & Nash-Sutcliffe Coefficient \\
\hline 1 & 175 & 0.61 \\
\hline 2 & 34 & 0.32 \\
\hline 3 & 221 & 0.18 \\
\hline 4 & 196 & 0.24 \\
\hline 5 & 81 & 0.60 \\
\hline 6 & 25 & 0.83 \\
\hline 7 & 210 & 0.72 \\
\hline 8 & 24 & 0.89 \\
\hline 9 & 155 & 0.38 \\
\hline 10 & 5 & 0.61 \\
\hline 11 & 4 & 0.13 \\
\hline 12 & 32 & 0.71 \\
\hline 13 & 248 & 0.51 \\
\hline 14 & 463 & 0.44 \\
\hline 15 & 61 & 0.22 \\
\hline 16 & 90 & 0.59 \\
\hline 17 & 29 & -0.23 \\
\hline
\end{tabular}


Table 9. Parameter values corresponding to global optimum; also shown are linear parameter confidence limits calculated as a by-product of the GML parameter estimation process.

\begin{tabular}{|l|l|l|l|}
\hline Parameter name & Estimated Value & $\begin{array}{l}\text { Lower 95 Percent } \\
\text { Confidence Limit }\end{array}$ & $\begin{array}{l}\text { Upper 95 Percent } \\
\text { Confidence Limit }\end{array}$ \\
\hline LENGTH & 0.816390 & 0.793602 & 0.839832 \\
\hline CONSTLOSS & 0.151830 & 0.143696 & 0.160425 \\
\hline SNYDERTP & 126.100 & 123.849 & 128.392 \\
\hline SNYDERCP & 0.100000 & $9.711740 E-02$ & 0.102968 \\
\hline GWSTOCOEFF & 1.87410 & 1.79730 & 1.95418 \\
\hline
\end{tabular}

Further evidence supporting the existence of local optima for the first HEC-HMS model was provided through optimization runs performed within HEC-HMS using the Nelder and Mead (1965) local search method and the sum of squared residuals objective function. Table 10 summarizes the results of the optimization runs.

Table 10. Initial parameter sets, final parameter sets, and objective function values associated with three HEC-HMS optimization runs with the first HEC-HMS model.

\begin{tabular}{|c|c|c|c|c|c|c|c|c|c|}
\hline \multirow[b]{3}{*}{ Parameter } & \multicolumn{9}{|c|}{ TRIAL } \\
\hline & \multicolumn{3}{|c|}{1} & \multicolumn{3}{|c|}{2} & \multicolumn{3}{|c|}{3} \\
\hline & $\begin{array}{c}\text { Initial Parameter } \\
\text { Value }\end{array}$ & $\begin{array}{l}\text { HMS Optimized } \\
\text { Param eter Value }\end{array}$ & $\begin{array}{c}\text { PD_MS2 } \\
\text { Optimized } \\
\text { Parameter Value }\end{array}$ & $\begin{array}{c}\text { Initial Parameter } \\
\text { Value }\end{array}$ & $\begin{array}{l}\text { HMS Optimized } \\
\text { Param eter Value }\end{array}$ & $\begin{array}{c}\text { PD_MS2 } \\
\text { Optimized } \\
\text { Parameter Value }\end{array}$ & $\begin{array}{l}\text { Initial Param eter } \\
\text { Value }\end{array}$ & $\begin{array}{l}\text { HMS Optimized } \\
\text { Parameter Value }\end{array}$ & $\begin{array}{c}\text { PD_MS2 } \\
\text { Optimized } \\
\text { Parameter Value }\end{array}$ \\
\hline Initial Deficit & 0.0063 & 0.1032 & 0.0005 & 3.9775 & 3.9775 & 0.0134 & 0.0667 & 0.1636 & 0.0130 \\
\hline Max Storage & 0.0137 & 0.4642 & 0.0010 & 5.0468 & 5.0468 & 0.0918 & 0.5799 & 1.0304 & 0.5673 \\
\hline Constant Loss & 6.9169 & 7.0139 & 1.6772 & 2.7432 & 2.7432 & 0.0416 & 8.5194 & 8.6164 & 8.5194 \\
\hline Snyder Peak & 385.27 & 385.37 & 500 & 0.2061 & 0.2061 & 0.2061 & 298.48 & 298.58 & 298.48 \\
\hline Snyder Coeff & 0.4062 & 0.5032 & 0.6882 & 0.7416 & 0.7416 & 0.7416 & 0.1470 & 0.2440 & 0.1470 \\
\hline GW Coeff & 1165.4 & 1165.5 & 853.75 & 9470.9 & 9470.9 & 11.83 & 9738.8 & 9738.9 & 8083.3 \\
\hline $\begin{array}{l}\text { HMS Objectlve } \\
\text { Function Value }\end{array}$ & & 49691.1000 & & & 50275.7000 & & & 50170.1000 & \\
\hline
\end{tabular}

Figure 10 is a plot of simulated flows and base flows at the global objective function minimum. While we are now able to identify the global objective function minimum using automated parameter estimation capabilities with a HEC-HMS model, this new capability does not preclude identification of a hydrologically unacceptable model at the global minimum. A more robust objective function formulation is needed that better reflects our perceptual model of the watershed system. 


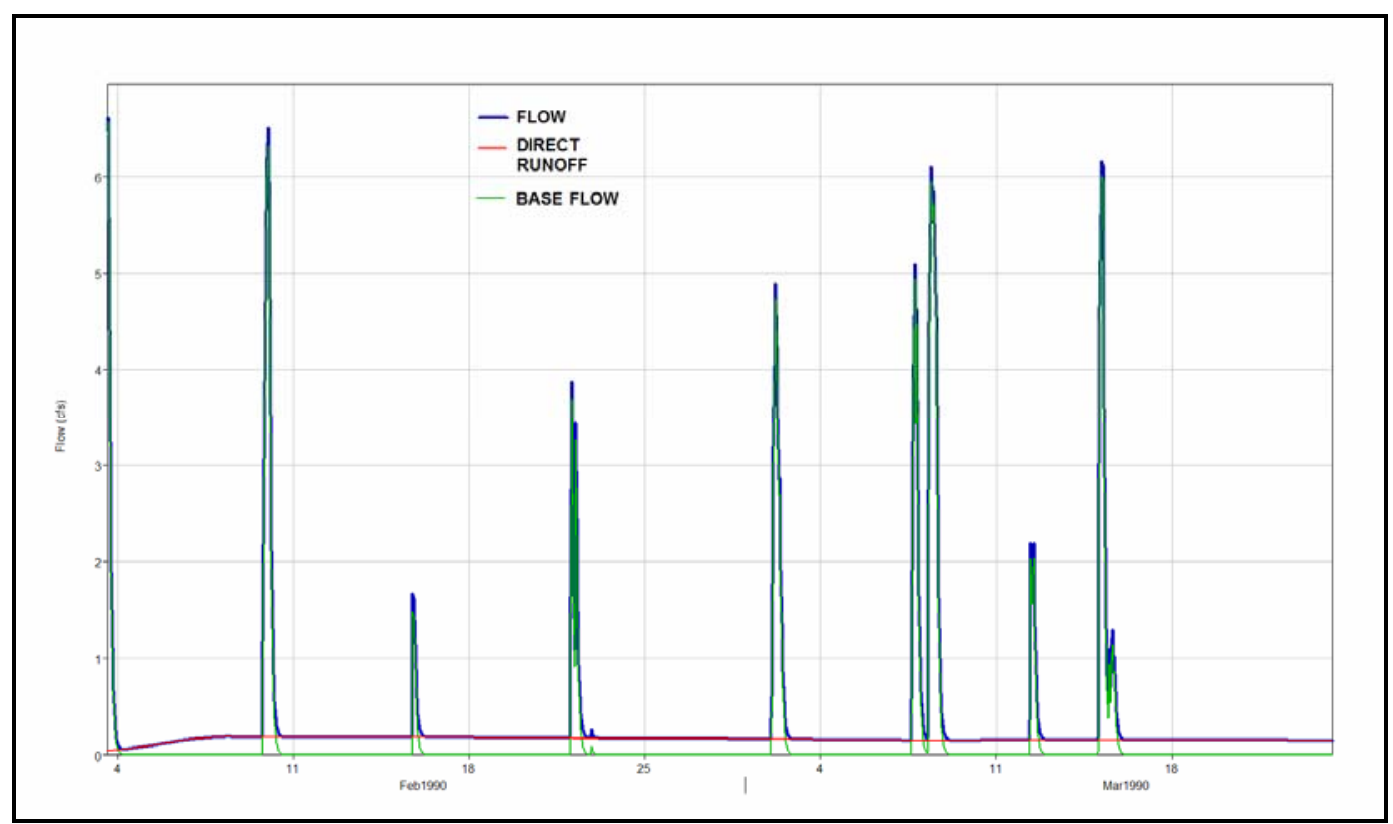

Figure 10. Plot of simulated flows, quickflow, and base flow at the global minimum.

\section{First HEC-HMS Model - Calibration Experiment 2}

In an attempt to locate as many local minima as possible, PD_MS2 was asked to run 100 inversion runs from a succession of starting values that were maximally distant from all previous parameter trajectories, as discussed above. These starting values were selected from 1,296 random parameter samples for which objective functions were calculated prior to the undertaking of any inversion runs. Figure 11 depicts the outcomes of this exercise.

For this calibration problem, the objective function has a value of 428.6 at its global minimum. Table 11 summarizes computed Nash-Sutcliffe efficiency scores (which are based on a comparison of the flow observations used in the calibration process) for each of the individual 17 noncontiguous time intervals. Figure 12 shows the fit between modeled and observed flows at this minimum for the flows that were compared for the seventh non-contiguous time interval listed in Table 2.

Figure 13 is a plot of the simulated flows and base flows at the global objective function minimum. While we have now demonstrated how state information other than stream discharge data alone can be included into the automatic calibration process of a HEC-HMS model, which results, at least for this case, in a much more hydrologically acceptable model than calibrating solely against flows, this process still may not remove the problem of local optima. 


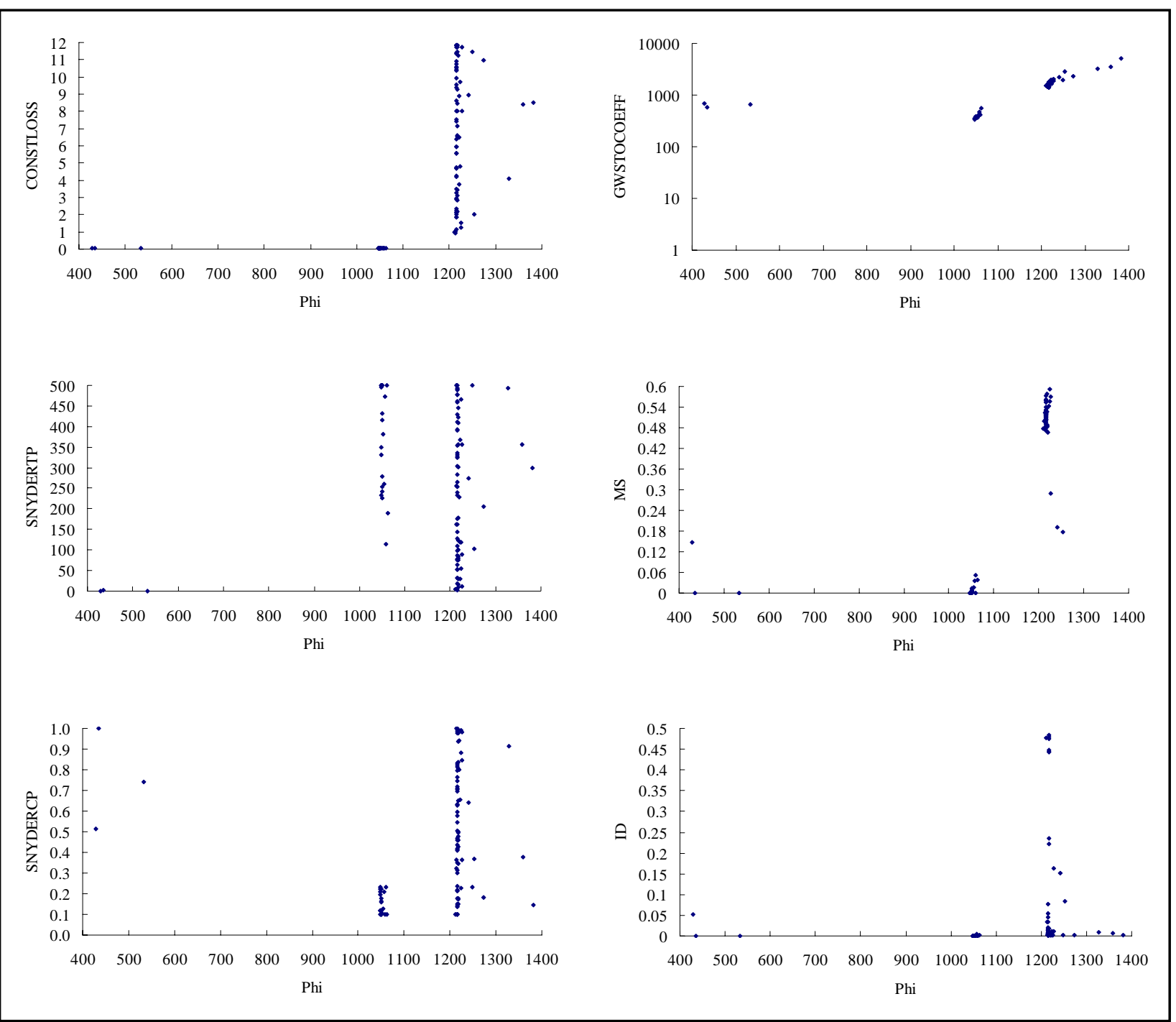

Figure 11. End-points in parameter space of 100 inversion runs undertaken under the control of PD_MS2. Parameters comprising an optimized set are linked vertically between graphs by objective function. TPI functionality was not operative. 
Table 11. Nash-Sutcliffe efficiency associated with identified global minimum.

\begin{tabular}{|l|l|l|}
\hline Number & Number of Series Terms in this Interval & Nash-Sutcliffe Coefficient \\
\hline 1 & 175 & 0.61 \\
\hline 2 & 34 & 0.70 \\
\hline 3 & 221 & 0.55 \\
\hline 4 & 196 & 0.51 \\
\hline 5 & 81 & 0.76 \\
\hline 6 & 25 & 0.09 \\
\hline 7 & 210 & 0.70 \\
\hline 8 & 24 & 0.73 \\
\hline 9 & 155 & 0.56 \\
\hline 10 & 5 & -14.18 \\
\hline 11 & 4 & -9.73 \\
\hline 12 & 32 & 0.34 \\
\hline 13 & 248 & 0.73 \\
\hline 14 & 463 & 0.74 \\
\hline 15 & 61 & -0.13 \\
\hline 16 & 90 & 0.33 \\
\hline 17 & 29 & -0.20 \\
\hline
\end{tabular}

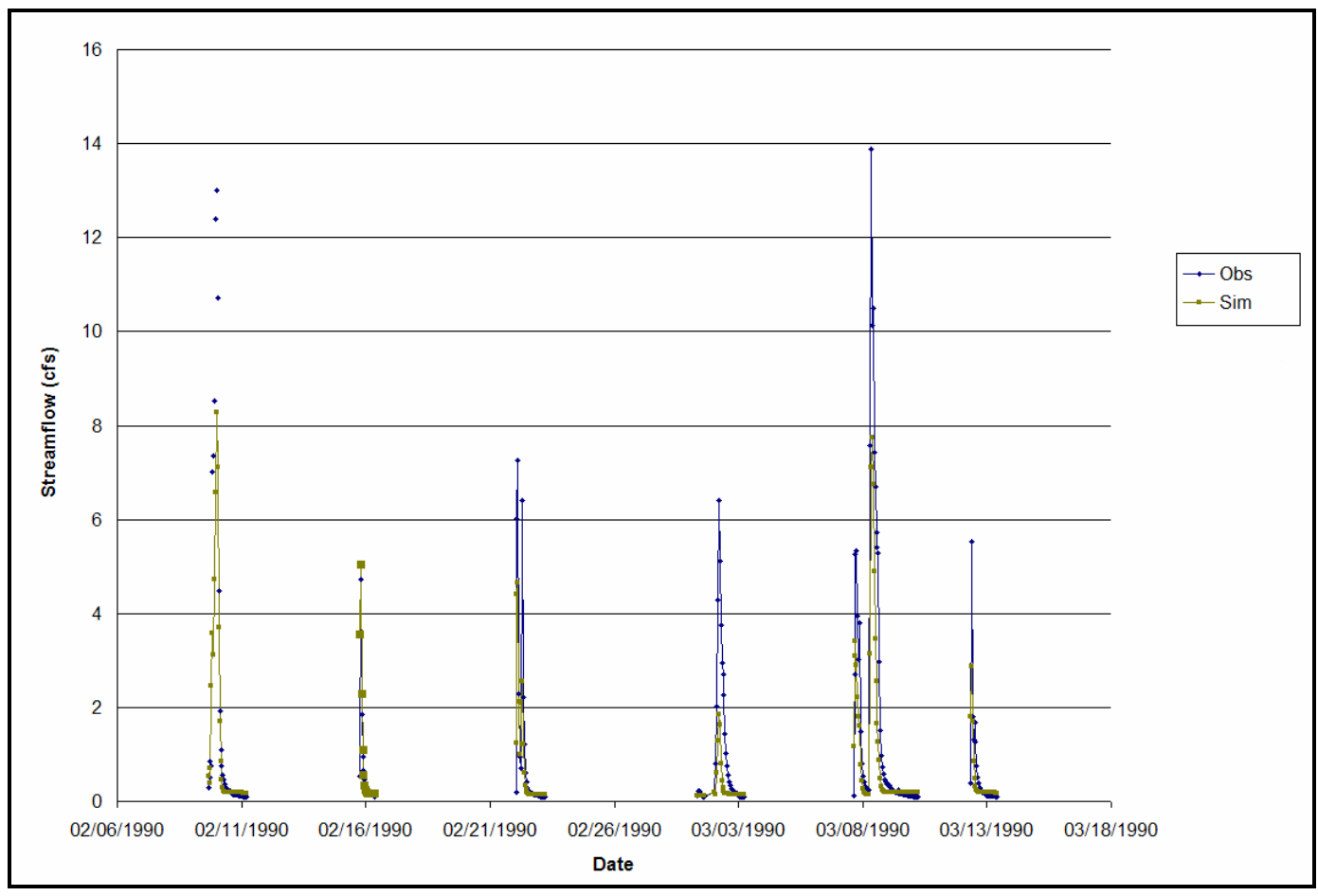

Figure 12. Simulated and observed flows at identified global minimum for the flows that were compared for the seventh non-contiguous time interval listed in Table 2. 


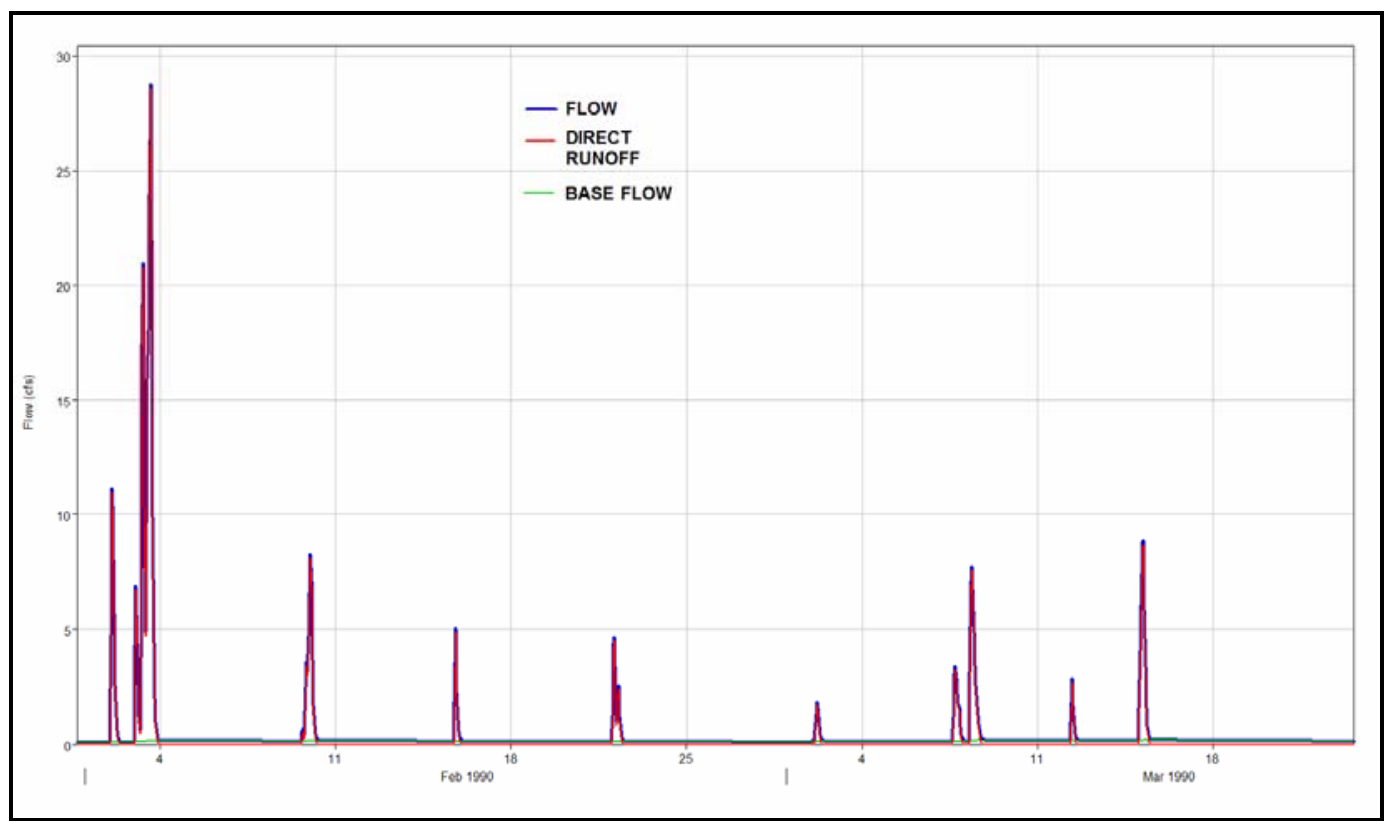

Figure 13. Plot of simulated flows, quickflow, and base flow at the global minimum.

\section{Second HEC-HMS Model}

Table 12 lists parameter values for each subwatershed model, estimated using the adaptive regularization scheme described above. In implementing the regularized inversion process, a very low target measurement objective function was set; hence $\Phi_{\mathrm{m}}$ of Equation 3 was lowered as far as possible, thus reducing misfit between measured and observed flows to a minimum. It is apparent from Table 12 that optimal fitting of model outputs to matched flows could only be achieved through the assignment of different values to parameters of the same type in different subwatersheds. However, the adaptive regularization scheme employed in their estimation attempted to ensure that these differences were kept to a minimum. The total measurement objective function (pertaining to all streamflow gauges) achieved through this calibration exercise was 1,047.

Table 13 summarizes computed Nash-Sutcliffe efficiency scores (which are based on a comparison of the flow observations used in the calibration process) for each of the individual non-contiguous time intervals for each gauged subwatershed.

Figure 14 shows the fit between the modeled and observed flows for streamflow gauging station 9 for the flows that were compared for the seventh non-contiguous time interval listed in Table 2. 
Table 12. Estimated values for subwatershed model parameters for attainment of best fit at all subwatershed streamflow gauging stations, this corresponding to a measurement objective function of 135.1. Adaptive regularization was employed in the parameter estimation process.

\begin{tabular}{|l|l|l|l|l|}
\hline Parameter Name & Station 8 & Station 9 & Station 11 & Station 12 \\
\hline MS & 1.031548 & 0.193263 & 0.169596 & 0.209377 \\
\hline ID & 0.143271 & 0.026842 & 0.023555 & 0.029080 \\
\hline CONSTLOSS & 0.024030 & 0.039661 & 0.080199 & 0.039745 \\
\hline SNYDERTP & 0.250000 & 0.250000 & 0.250000 & 0.250000 \\
\hline SNYDERCP & 0.560000 & 0.560000 & 0.560000 & 0.560000 \\
\hline GWSTOCOEFF & 12.316100 & 16.01440 & 11.648300 & 9.545690 \\
\hline
\end{tabular}

Table 13. Number of terms and Nash-Sutcliffe efficiency scores, ES, associated with identified minimum for each station.

\begin{tabular}{|c|c|c|c|c|c|c|c|c|}
\hline \multirow[b]{3}{*}{ Number } & \multicolumn{8}{|c|}{ Station } \\
\hline & \multicolumn{2}{|c|}{8} & \multicolumn{2}{|c|}{9} & \multicolumn{2}{|r|}{11} & \multicolumn{2}{|r|}{12} \\
\hline & $\begin{array}{l}\text { Measure- } \\
\text { ment }\end{array}$ & ES & $\begin{array}{l}\text { Measure- } \\
\text { ment }\end{array}$ & ES & $\begin{array}{l}\text { Measure- } \\
\text { ment }\end{array}$ & ES & $\begin{array}{l}\text { Measure- } \\
\text { ment }\end{array}$ & ES \\
\hline 1 & 29 & 0.76 & 175 & 0.79 & 45 & 0.85 & 58 & 0.74 \\
\hline 2 & 69 & -2.35 & 34 & 0.82 & 306 & 0.17 & 306 & 0.17 \\
\hline 3 & 237 & 0.53 & 221 & 0.69 & 84 & 0.75 & 84 & 0.75 \\
\hline 4 & 18 & 0.35 & 196 & 0.69 & 215 & -0.04 & 356 & 0.12 \\
\hline 5 & 25 & -18.92 & 81 & 0.69 & 249 & 0.86 & 334 & 0.86 \\
\hline 6 & 95 & -0.28 & 25 & 0.50 & 104 & 0.64 & 104 & 0.64 \\
\hline 7 & 288 & 0.66 & 210 & 0.82 & 63 & 0.68 & 58 & 0.68 \\
\hline 8 & 393 & 0.81 & 24 & 0.66 & 123 & 0.46 & 123 & 0.46 \\
\hline 9 & 124 & 0.28 & 155 & 0.62 & 94 & 0.36 & 94 & 0.36 \\
\hline 10 & 115 & 0.81 & 5 & -20.57 & 45 & -2.66 & 45 & -2.66 \\
\hline 11 & 101 & 0.66 & 4 & -12.35 & 27 & -13.11 & 27 & -13.11 \\
\hline 12 & 26 & 0.75 & & & 132 & 0.63 & 132 & 0.63 \\
\hline 13 & 69 & -2.35 & & & 208 & 0.86 & 142 & 0.86 \\
\hline 14 & 237 & 0.53 & & & 95 & 0.72 & 95 & 0.72 \\
\hline 15 & 14 & 0.27 & & & 92 & -1.52 & 65 & -2.07 \\
\hline 16 & 24 & -19.59 & & & 111 & -0.30 & 111 & -0.30 \\
\hline 17 & 95 & -0.28 & & & 29 & -12.04 & 29 & -12.04 \\
\hline 18 & 248 & 0.66 & & & 20 & -4.26 & 14 & -5.09 \\
\hline 19 & 327 & 0.81 & & & 158 & 0.52 & & \\
\hline 20 & 108 & 0.26 & & & 45 & & & \\
\hline 21 & 129 & 0.81 & & & 306 & & & \\
\hline 22 & 101 & 0.66 & & & 84 & & & \\
\hline 23 & 101 & 0.66 & & & 215 & & & \\
\hline
\end{tabular}




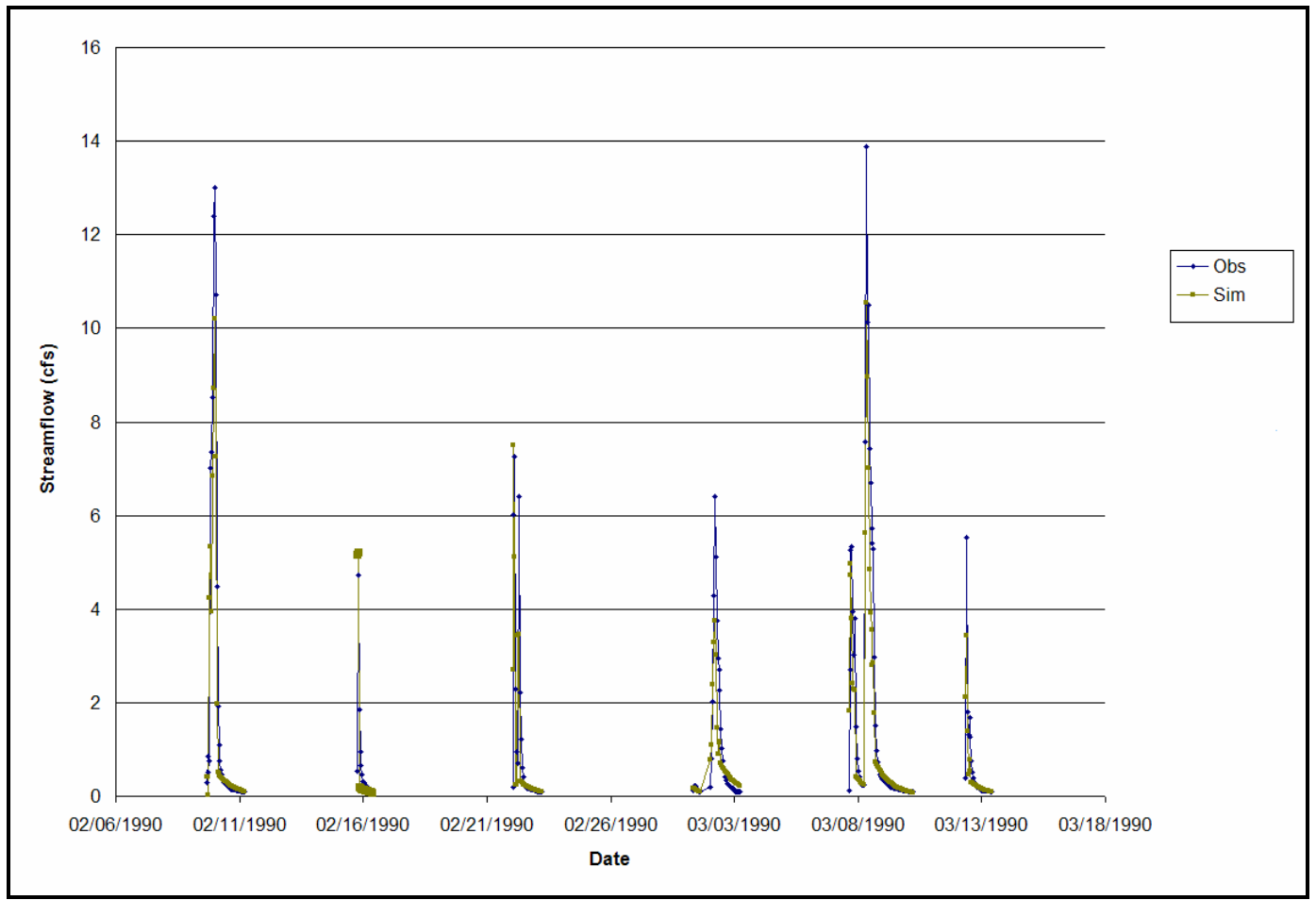

Figure 14. Simulated and observed flows at station 9 , at the minimum objective function value, for the flows that were compared for the seventh non-contiguous time interval listed in Table 2.

Figure 15 is a plot of the simulated flows and base flows for streamflow gauging station 9 at the objective function minimum.

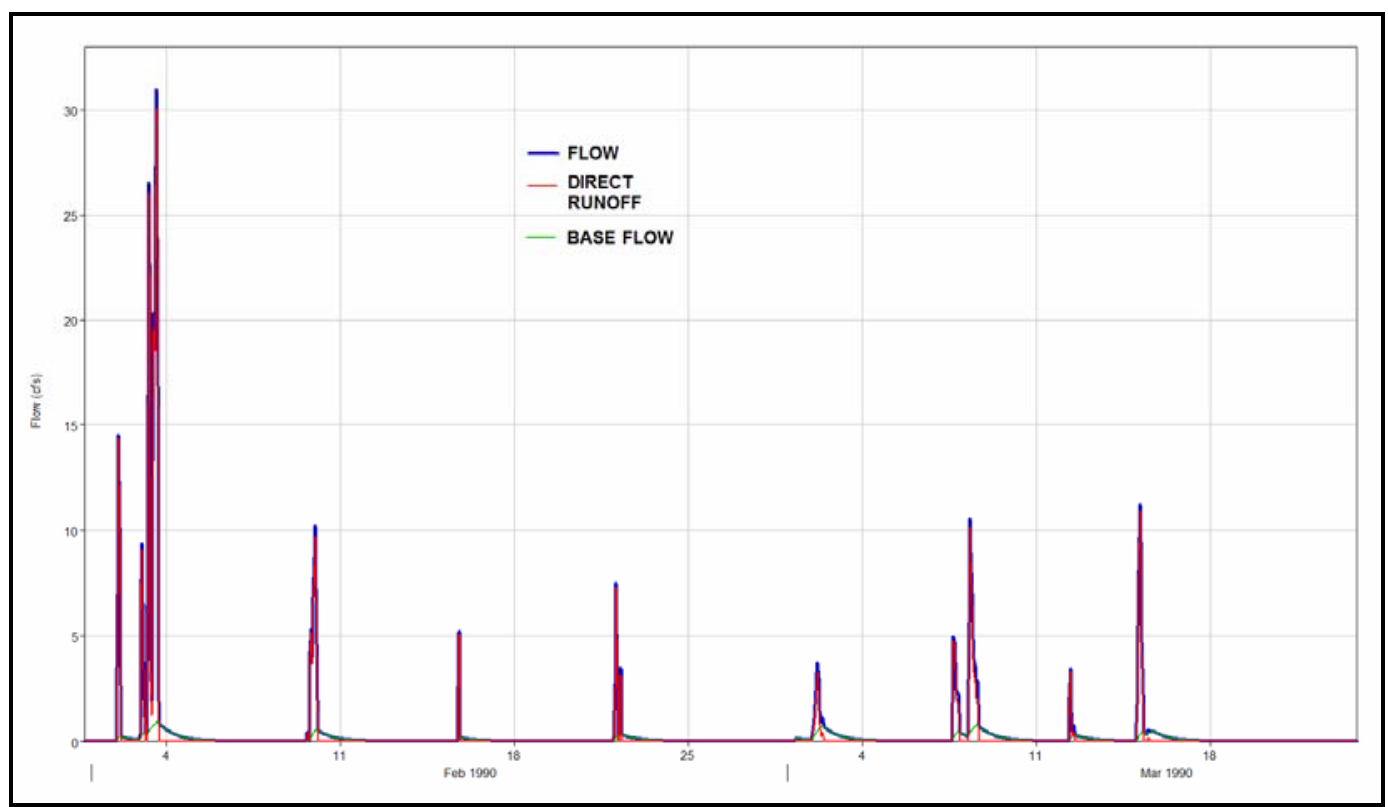

Figure 15. Plot of simulated flows, quickflow, and base flow, for station 9, at the minimum. 


\section{Discussion}

The objectives for this article were to describe and demonstrate the use of parameter estimation methodologies that could potentially be employed to improve upon existing HEC-HMS (Hydrologic Engineering Center's Hydrologic Modeling System) automated parameter estimation capabilities. In particular, the intent for this article was to describe and demonstrate the use of methods that (1) accommodate local minima (Skahill and Doherty 2006) and (2) support the inversion of complex (i.e., highly parameterized) models (Doherty and Skahill 2006), and through this process to also:

a. Demonstrate that each of these two methods provide information about individual parameter sensitivities and parameter correlation.

b. Demonstrate how state information other than stream discharge data alone can be included into the automatic calibration process of an HEC-HMS model.

c. Demonstrate how objective function definitions associated with specific periods of the calibration dataset can be included into the automatic calibration process of an HEC-HMS model.

The principal intent of the first calibration experiment with the first HECHMS model was to illustrate the existence of multiple local optima (and this was also demonstrated through the trials using the automatic calibration capabilities currently available within HEC-HMS), and to also demonstrate that the trajectory repulsion scheme can be used with HEC-HMS to accommodate the presence of multiple local optima and find the global objective function minimum.

The initial deficit parameter of the deficit constant loss model (HEC 2005) was identified to be completely insensitive at the identified global objective function minimum for the first HEC-HMS model. Estimates of parameter uncertainty, correlation and (in)sensitivity are readily available as a byproduct of the use, both during and at the end of a model inversion, of the parameter estimation methods described in the theory section of this article, and such information can be readily employed to reformulate a 
problem to achieve a more stable inversion and likely also a more optimal model estimate.

Simulated flows from the estimated model at the global objective function minimum for the first calibration experiment with the first HEC-HMS model fit observed stream discharges fairly well; however, upon further inspection it was an unacceptable model in that almost all simulated flow was base flow (see Figure 10). This contradicted our perceptual model for the watershed system, and past research, wherein system response is dominated by direct runoff. This observation underscores the fact that the ability to find the global objective function minimum is an insufficient requirement to attain a hydrologically acceptable model. If at all possible, one must process the observation dataset(s) in a manner that best reflects our perceptual understanding of a system's hydrologic response.

The principal intent of the second calibration experiment with the first HEC-HMS model was to demonstrate that state information other than stream discharge data alone can be included into the automatic calibration process of an HEC-HMS model, and through that process, at least for this case, one can attain a more hydrologically acceptable model than calibrating solely against flows (see Figure 13), and moreover, retain a reasonable fit with the actual hard data (see Tables 8 and 11 and Figures 9 and 12). However, as evidenced by inspecting Figure 11, this process does not circumvent the presence of multiple local optima.

The calibration experiment with the second HEC-HMS model demonstrated that one can simultaneously calibrate multiple subwatershed models automatically with an HEC-HMS model. The strength of such an approach is that it includes into the calibration process explicit recognition of the fact that the semi-physical basis of parameters employed by models such as HEC-HMS demands that parameter values associated with similar land uses and soil types in adjacent areas be at least broadly similar. Manual calibration of the four Goodwin Creek subwatershed models, if carefully implemented, would probably follow this strategy (i.e., calibrate each model individually, with due recognition of the desirability of intersubwatershed parameter similarity), for a modeler would be aware of the need for maintaining at least a certain degree of parameter similarity across subwatersheds as he/ she assigns values to them in successive trialand-error model runs. However, it is likely that this would be a difficult undertaking from a practical point of view, probably requiring much pa- 
tience on the part of the modeler, while many model runs are undertaken to achieve this ideal. Furthermore such an exercise would leave the modeler with no knowledge of whether model-to-measurement fits on the one hand, and inter-subwatershed parameter similarity on the other hand, could be further improved with an even greater amount of time devoted to the manual calibration exercise. Hence, computer assistance in this process is obviously desirable. However, the use of automated methods of model calibration must not reduce the capacity of the modeler to exercise his/ her judgment in this and other matters; in fact, it should enhance it. Fortunately, regularized inversion does indeed allow a modeler's judgment to become an integral part of the calibration process through implementing that process as a constrained minimization problem, with the constraints being the modeler's idea of what constitutes an ideal parameter set. Departures from these constraints are tolerable only to the extent that they result in an "adequate" (as defined by the modeler) level of model-tomeasurement fit.

For the Goodwin Creek Experimental Watershed, inclusion of a modeler's wisdom in the calibration process possibly assumes more importance than in many other modeling contexts because of the potential paucity of data available for the calibration of each individual subwatershed, and hence the high potential for parameter nonuniqueness. Simultaneous calibration of four subwatershed models allows the quantity of data employed by the parameter estimation process to be increased by a factor of four. In theory, this provides some relief from the effects of data paucity in estimating parameters for four individual subwatershed models through separate calibration exercises. This is because it does this without necessarily introducing four times the number of parameters to the calibration process, for the constrained optimization process implemented through regularized inversion dispenses with the need for parameters in different subwatersheds to vary independently of each other unless the information content of the calibration dataset makes such parameter differences tenable. Where a certain degree of parameter independence is proven to be warranted, extra "observations" are introduced to the parameter estimation process through the regularization algorithm through which this process is implemented - "observations" whose presence should endow that process with extra numerical stability because of the fact that they pertain directly to model parameters themselves, and that at least one such "observation" exists for every estimable parameter. In theory, the result is a stable process that allows maximum receptivity of parameters to both "hard information" 
provided by the measurement dataset and "soft information" embodied in a modeler's understanding of the area, encapsulated in the set of regularization constraints. The results shown in Table 13 (in comparison to Tables 8 and 11) and Figures 14 and 15 seem to confirm the above noted comments.

All three calibration experiments demonstrated how objective function definitions associated with specific periods of the calibration dataset can be included into the automatic calibration process of an HEC-HMS model, and this was achieved due to the model independent nature of the methods described.

While the PD_MS2 "multi-start" procedure is smart in that it uses the trajectories from previous local searches to select new initial conditions that are maximally distant in parameter space, it is nevertheless inefficient in that it explores many parts of parameter space which are dead ends (and this is clearly evident upon inspection of Figures 8 and 11). Hence, future work will focus on the selection, adaptation, and implementation of a hybrid genetic and gradient-based optimization algorithm for identifying globally optimal model parameters.

Further research is also needed to address proper objective function formulation, and weights assignment, to ensure that the model identified at the conclusion of an estimation process is reflective of the multiple processes (which operate at different time scales) encapsulated in the model structure, intended uses for the model, and the data available to identify the parameters related to those processes and intended predictions. Another avenue for investigation will be the use of multi-resolution analysis (wavelets) to decompose the observed system response data into different time scales to reflect the time scales of differing processes in the watershed model.

Further research will also explore the use of additional regularization strategies. Utilization of the time-scale decomposed objective function will allow regularization strategies that reflect the physical time scales present in the model and observed response data. 


\section{References}

Aster, R. C., B. Borchers, and C. H. Thurber. 2005. Parameter estimation and inverse problems. Elsevier, Inc., $301 \mathrm{p}$.

Bard, J . 1974. Nonlinear parameter estimation. NY: Academic Press, 341 p.

Bates, B. C., and E. P. Campbell. 2001. A Markov chain Monte Carlo scheme for parameter estimation and inference in conceptual rainfall-runoff modeling. Water Resour. Res. 37 (4): 937-947.

Beven, K. 2001. Rainfall-runoff modelling: The primer. NY: J ohn Wiley \& Sons, 360 p.

Blackmarr, W. A. (1995). Documentation of hydrologic, geomorphic, and sediment transport measurements on the Goodwin Creek Experimental Watershed, Northern Mississippi, for the period 1982-1993 - Preliminary release. Research Report No. 3 (CD-ROM). U.S. Dept. of Agriculture. Agric. Research Service.

Box, G. E. P., and D. R. Cox. 1964. An analysis of transformations. J .R. Stat. Soc. Ser. B 26: 211-243.

Box, G. E. P., and G. M. J enkins. 1976. Time series analysis: Forecasting and control. San Francisco: Holden-Day.

Box, G. E. P., and G. C. Tiao. 1973. Bayesian inference in statistical analysis. Reading, MA: Addison-Wesley.

Boyle, D. P., Gupta, H. V., and S. Sorooshian. 2000. Toward improved calibration of hydrologic models: combining the strengths of manual and automatic methods. Water Resour. Res. 36(12): 3663-3674.

Conte, S. D., and C. de Boor. 1972. Elementary numerical analysis. NY: MoGraw-Hill.

Doherty, J., and J . M. J ohnston. 2003. Methodologies for calibration and predictive analysis of a watershed model. J . American Water Resources Association 39(2): 251-265.

Doherty, J ., and B. Skahill. 2006. An advanced regularization methodology for use in watershed model calibration. Accepted for publication in J ournal of Hydrology.

Downer, C. W., and F. L. Ogden. 2003. Prediction of runoff and soil moistures at the watershed scale: Effects of model complexity and parameter assignment. Water Resour. Res. 39(3): 1045.

Duan, Q. S., S. Sorooshian, and V. K. Gupta. 1992. Effective and efficient global optimization for conceptual rainfall runoff models. Water Resour. Res. 28 (4): 1015-1031.

Gupta, H. V., S. Sorooshian, T. S. Hogue, and D. P. Boyle. 2003. Advances in automatic calibration of watershed models. In Water Science and Application Series ed. Q. Duan, H. Gupta, S. Sorooshian, A. Rousseau, and R. Turcotte. 6: 197-211. 
Hydrologic Engineering Center. 2005. Hydrologic modeling system HEC-HMS: User's manual. Version 3.0.0. Davis, CA: U.S. Army Corps of Engineers.

Hydrologic Engineering Center. 2000. Hydrologic modeling system HEC-HMS: Technical reference manual. Davis, CA: U.S. Army Corps of Engineers.

Kuczera, G. 1983. Improved parameter inference in catchment models. 1. Evaluating parameter uncertainty. Water Resour. Res. 19 (5): 1151-1172.

Levenberg, K. 1944. A method for the solution of certain problems in least squares. Q. Appl. Math. 2: 164-168.

Marquardt, D. 1963. An algorithm for least-squares estimation of non-linear parameters. SIAMJ . Appl. Math. 11: 431-441.

Madsen, H. 2000. Automatic calibration of a conceptual rainfall-runoff model using multiple objectives. J ournal of Hydrology 235: 276-288.

Moore, C., and J . Doherty. 2005. The role of the calibration process in reducing model predictive error. Water Resources Research 41(5): W05020.

Nathan, R. J., and T. A. McMahon. 1990. Evaluation of automated base flow and recession analyses. Water Resources Research 26(7): 1465-1473.

Nelder, J . A., and R. Mead. 1965. A simplex method for function minimization. Computer Journal 7: 308-313.

Skahill, B. 2006. A model independent parameter estimation toolbox: Version 1. U.S. Army Engineer Research and Development Center Technical Report. In preparation.

Skahill, B., and J . Doherty. 2006. Efficient accommodation of local minima in watershed model calibration. Accepted for publication in J ournal of Hydrology.

Vecchia, A. V., and R. L. Cooley. 1987. Simultaneous confidence and prediction intervals for nonlinear regression models with application to a groundwater flow model. Water Resour. Res. 23(7): 1237-1250.

Wagener, T., H. S. Wheater, and H. V. Gupta. 2004. Rainfall-runoff modelling in gauged and ungauged catchments. London, UK: Imperial College Press, 300 p. 


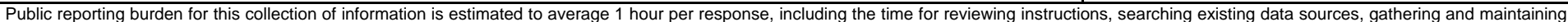

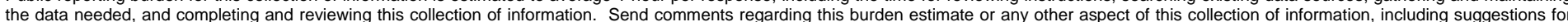

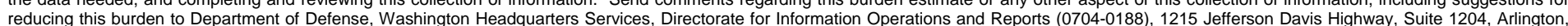

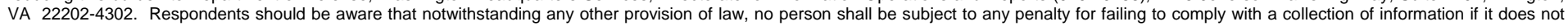
display a currently valid OMB control number. PLEASE DO NOT RETURN YOUR FORM TO THE ABOVE ADDRESS.

\begin{tabular}{l|c}
$\begin{array}{l}\text { 1. REPORT DATE (DD-MM-YYYY) } \\
\text { August } 2006\end{array}$ & $\begin{array}{c}\text { 2. REPORT TYPE } \\
\text { Final report }\end{array}$ \\
\hline
\end{tabular}

\section{TITLE AND SUBTITLE}

Potential Improvements for HEC-HMS Automated Parameter Estimation

6. AUTHOR(S)

Brian E. Skahill
3. DATES COVERED (From - To)

5a. CONTRACT NUMBER

5b. GRANT NUMBER

5c. PROGRAM ELEMENT NUMBER

5d. PROJECT NUMBER

5e. TASK NUMBER

5f. WORK UNIT NUMBER

8. PERFORMING ORGANIZATION REPORT NUMBER

ERDC/CHL TR-06-13

10. SPONSOR/MONITOR'S ACRONYM(S)

11. SPONSOR/MONITOR'S REPORT NUMBER(S)

\section{DISTRIBUTION / AVAILABILITY STATEMENT}

Approved for public release; distribution is unlimited.

\section{SUPPLEMENTARY NOTES}

\section{ABSTRACT}

Model independent enhancements and adaptations to the Gauss-Marquardt-Levenberg (GML) method of computer-based parameter estimation are described and demonstrated as potential improvements to existing HEC-HMS automatic calibration capabilities. In contrast to existing HEC-HMS automated parameter estimation capabilities, these methods support global optimization, the ability to simultaneously calibrate multiple subwatershed systems represented within an HEC-HMS model, and they also provide information about individual parameter sensitivities and parameter correlation during and at the end of the calibration process. Moreover, their model independent nature allows one to include into the calibration process (1) state information other than simply stream discharge data, (2) multiple periods, rather than a single time window, of the calibration dataset(s), and (3) the ability to weight data in order to accommodate a prediction specific calibration effort or to accommodate suspect and/or missing observations. The methods are demonstrated by calibrating HEC-HMS models to subwatershed systems in the Goodwin Creek Experimental Watershed.

\section{SUBJECT TERMS}

HEC-HMS

Watershed Model Calibration

16. SECURITY CLASSIFICATION OF:

\begin{tabular}{|l|l|}
\hline a. REPORT & b. ABSTRACT \\
UNCLASSIFIED & UNCLASSIFIED \\
\hline
\end{tabular}

Automatic Parameter Estimation Objective Function Local Minima

\begin{tabular}{|l|c|c|}
\multicolumn{2}{|l|}{$\begin{array}{l}\text { 17. LIMITATION } \\
\text { OF ABSTRACT } \\
\text { UNCLASSIFIED }\end{array}$} & $\begin{array}{c}\text { 18. NUMBER } \\
\text { OF PAGES }\end{array}$ \\
\cline { 1 - 1 } & & 60 \\
\hline
\end{tabular}

\section{Regularization}

19a. NAME OF RESPONSIBLE PERSON

19b. TELEPHONE NUMBER (include area code) 